\title{
Terrestrial Planet Formation in a protoplanetary disk with a local mass depletion: A successful scenario for the formation of Mars
}

\author{
A. Izidoro ${ }^{1,2,3}$ \\ izidoro@feg.unesp.br \\ N. Haghighipour ${ }^{4,5}$ \\ nader@ifa.hawaii.edu \\ O. C. Winter ${ }^{1}$ \\ and \\ M. Tsuchida ${ }^{6}$
}

\begin{abstract}
Models of terrestrial planet formation for our solar system have been successful in producing planets with masses and orbits similar to those of Venus and Earth. However, these models have generally failed to produce Mars-sized objects around 1.5 AU. The body that is usually formed around Mars' semimajor axis is, in general, much more massive than Mars. Only when Jupiter and Saturn are assumed to have initially very eccentric orbits $(\mathrm{e} \sim 0.1)$, which seems fairly unlikely for the solar system, or alternately, if the protoplanetary disk is truncated at 1.0 AU, simulations have been able to produce Mars-like bodies in the correct location. In this paper, we examine an alternative scenario for the
\end{abstract}

\footnotetext{
1 UNESP, Univ. Estadual Paulista - Grupo de Dinâmica Orbital \& Planetologia, Guaratinguetá, CEP 12.516-410, São Paulo, Brazil

${ }^{2}$ Capes Foundation, Ministry of Education of Brazil, Brasília/DF 70040-020, Brazil.

${ }^{3}$ University of Nice-Sophia Antipolis, CNRS, Observatoire de la Côte d'Azur, Laboratoire Lagrange, BP 4229, 06304 Nice Cedex 4, France.

${ }^{4}$ Institute for Astronomy and NASA Astrobiology Institute, University of Hawaii-Manoa, Honolulu, HI 96822, USA

${ }^{5}$ Institute for Astronomy and Astrophysics, University of Tübingen, 72076 Tübingen, Germany

${ }^{6}$ UNESP, Univ. Estadual Paulista, DCCE-IBILCE, São José do Rio Preto, CEP 15.054-000, São Paulo, Brazil
} 
formation of Mars in which a local depletion in the density of the protosolar nebula results in a non-uniform formation of planetary embryos and ultimately the formation of Mars-sized planets around 1.5 AU. We have carried out extensive numerical simulations of the formation of terrestrial planets in such a disk for different scales of the local density depletion, and for different orbital configurations of the giant planets. Our simulations point to the possibility of the formation of Mars-sized bodies around 1.5 AU, specifically when the scale of the disk local mass-depletion is moderately high (50-75\%) and Jupiter and Saturn are initially in their current orbits. In these systems, Mars-analogs are formed from the protoplanetary materials that originate in the regions of disk interior or exterior to the local mass-depletion. Results also indicate that Earth-sized planets can form around $1 \mathrm{AU}$ with a substantial amount of water accreted via primitive water-rich planetesimals and planetary embryos. We present the results of our study and discuss their implications for the formation of terrestrial planets in our solar system.

Subject headings: Planets and satellites: formation; Methods: numerical

\section{Introduction}

A major obstacle in developing a comprehensive model for the formation of the terrestrial planets of our solar system is the planet Mars. Despite more than two decades of efforts in explaining the formation of the inner solar system,(e.g., Chambers \& Wetherill 1998; Agnor et al. 1999; Chambers 2001; Chambers \& Wetherill 2001), and many sophisticated and high resolution computational simulations of the late stage of terrestrial planet formation (Raymond et al., 2004, 2006; O'Brien et al. 2006; Raymond et al. 2007, 2009), the formation of Mars is still a mystery. While modern simulations have been successful in producing a wide variety of terrestrial planets such as two to four planets in well-separated and stable orbits (Raymond et al., 2004, 2006; O'Brien et al. 2006; Raymond et al. 2007, 2009; Lykawka \& Ito, 2013) and have been able to account for the origin of Earth's water and its accretion within the timescale consistent with radioactive chronometers (Allégre et al. 1995; Yin et al. 2002; Touboul et al. 2007; Marty 2012), they have not been able to form a Mars-analog at 1.5 AU. The body that is produced around the current Mars' semimajor axis is, in general, too massive when compared to the mass of Mars (See Raymond et. al., 2009 and the review by Morbidelli et al. 2012).

An important factor in determining the outcome of the simulations of terrestrial planet

formation is the orbital configuration of giant planets. As shown by many authors, the 
mean-motion and secular resonances due to these objects strongly affect the dynamics of planetesimals and protoplanetary bodies which play a significant role in their radial mixing and may also result in their ejection from the system (Chambers 2001; Chambers \& Cassen 2002; Levison \& Agnor 2003; Raymond et al. 2004, 2006, 2009; Agnor \& Lin 2012; Haghighipour et al. 2013). Regarding the formation of Mars, Mars-analogs with appropriate masses have only been produced with Jupiter and Saturn initially in relatively eccentric orbits (e.g., $e_{\text {Jup }}=e_{\text {Sat }}=0.1$, Thommes et al. 2008, Raymond et. al. 2009).

Although the current orbits of Jupiter and Saturn do not carry high eccentricities, early orbital instabilities among these planets (before the beginning of the late stage of terrestrial planet formation) could have increased their eccentricities to high values (Lega et al., 2013). While such an increase in orbital eccentricity is a natural consequence of planets' instability, the damping of these eccentricities to their present values, and ensuring that the outer solar system would in fact develop and maintain its current features, has proven to be a difficult task (Raymond et al. 2009). The high eccentricities of Jupiter and Saturn will also affect the process of the delivery of water-rich planetesimals and planetary embryos to the accretion zone of Earth, causing many of these objects to be scattered out of the system, leaving Earth with less water-carrying material to accrete. Models of the origin of Earth's water suggest that a significant fraction of Earth's water was delivered to its accretion zone by water-rich planetesimals and planetary embryos from past 2 AU (Morbidelli et al., 2000; Raymond et al., 2004; 2006; 2007; 2009; O’Brien et al., 2006; Izidoro et al., 2013). An Eccentric Jupiter and Saturn will very quickly deplete the protoplanetary disk inside Jupiter's orbit from its water-carrying objects and causes planets formed around Earth's semimajor axis to have much less water than expected (Raymond et al., 2009).

To overcome the difficulties of forming a Mars-sized planet at 1.5 AU, Hansen (2009) proposed that terrestrial planets might have formed in a narrow annulus of protoplanetary bodies around $1 \mathrm{AU}$. The results of the simulations by this author showed that they can successfully form Venus, Earth and Mars-analogs in a timescale consistent with isotopic constrains [see Kleine et al. (2009) for the constraints on the time of the formation of Earth, and Dauphas \& Pourmand (2011) for the constraints on the time of the formation of Mars]. The small mass of Mars in this model is the natural consequence of its accretion from the material in the edge of the protoplanetary disk, where it is subsequently scattered out of the annulus and isolated from other planetary embryos, keeping a low-mass while other planetary embryos grow. It is important to note that the formation of Mercury-analog planets is still an issue in the models of terrestrial planet formation and was only studied in a very limited sample of the simulations presented in Hansen (2009).

The success of the model by Hansen (2009) points to an interesting pathway for the 
formation of terrestrial planets. This model requires as much as $2 M_{\oplus}$ of material to be confined in the region between $0.7 \mathrm{AU}$ and 1.0 AU (equivalent to a disk with 3 times the minimum mass solar nebula) implying that a model for the formation of the planets in the inner solar system has to be able to present a scenario through which such a large amount of mass is confined in a small region. Recently Walsh et al (2011) proposed that it would be possible to create a narrow annulus of mass around $1 \mathrm{AU}$ and also deliver water-carrying objects to the accretion zone of Earth, a topic that is not considered by Hansen (2009), if one allows for Jupiter and Saturn to migrate inward from their regions of formation to $\sim 1.5$ $\mathrm{AU}$ at which point these planets change direction and migrate outward to a region close to their current orbits. In this model, the delivery of water-rich planetesimals and planetary embryos to the terrestrial zone is a natural consequence of the outward migration of giant planets. Known as the "Grand Tack" model, this scenario has been able to provide the region of terrestrial planets with the necessary amount of mass prior to the onset of their formation.

Although the Grand Tack model has been able to produce Mars-analogs and account for the current architecture of the asteroid belt, it may not agree entirely with the models of the formation and migration of giant planets. Hydrodynamical simulations show that the migration in a protoplanetary disk is very sensitive to numerous disk parameters (Morbidelli \& Crida, 2007) which are hard to constrain. In order to assess the validity of the GrandTack scenario on the formation and migration of Jupiter and Saturn, Pierens \& Raymond (2011) simulated the growth of these planets and their dynamical evolution driven by tidal interactions with a gas-dominated disk. The results of simulations by these authors showed that the two-phase migration of giant planets could be a natural outcome of the solar system evolution, probably coinciding with the late phases of the dissipation of the solar nebula. In a recent study of the migration of giant planets in an evolved gaseous disk, however, D'angelo \& Marzari (2012) have shown that only under favorable conditions and for a small region of the parameter space, the outward migration of Jupiter and Saturn can reach beyond 5 AU (as proposed by the Grand-Tack Model). It is important to note that if the outward migration of Jupiter and Saturn is not sufficiently fast, the efficiency of the Grand-Tack model decreases. The fast inward-then-outward migration of Jupiter and Saturn is the key to reproducing the architecture of the asteroid belt, with two distinct populations corresponding to the Cand S-type asteroids. Also, if the two-phase migration and its change of direction is not fast enough, the protoplanetary bodies in terrestrial planet region will be subject to the gravitational perturbation of giant planets for a long time which may result in their ejection from that region. The latter may subsequently reduce the mass in the planet-forming annulus to below its critical value disrupting the formation of terrestrial planets in that region.

A planet-forming disk is a complex and dynamic environment. During the course of 
planet formation, beginning from the stage when the dust particles coagulate to when the cores of giant planets are formed and terrestrial planets began their accretion, the physical properties of the disk are continuously subject to change. For instance, regions may appear where the disk is turbulent, or at places the density of the gas or solid material may be temporarily enhanced (e.g., Chiang \& Goldreich 1997; Papaloizou \& Nelson 2003; Laughlin et al. 2004; Garaud \& Lin 2007). These processes affect the radial profile and the physical properties of the disk (e.g., temperature and surface density), and may deviate them from simple power-laws. As proposed by Jin et al. (2008), the radial variations in the ionization fraction of the disk, for instance, can separate the disk into an inner high-viscosity part and an outer low-viscosity region. The difference in viscosities in these two regions causes the material in the inner part of the disk to flow faster toward the star compared to the material in the outer part, creating a local minimum in the disk's mass distribution in the boundary between these two regions. As shown by these authors, during the evolution of the nebula around the Sun, two of such regions might have appeared. One region is narrow with a width of approximately $0.1 \mathrm{AU}$, varying between $1.3 \mathrm{AU}$ and $3.4 \mathrm{AU}$, with a most probable location in the range of 1.3-2.4 AU. The second region is larger, with a width of $\sim 1 \mathrm{AU}$ centered at about 1.6 AU. Jin et al. (2008) suggested that the appearance of such a local minimum in the disk could result in a non-uniform formation and distribution of planetary embryos around the orbit of Mars, and will ultimately result in the formation of Mars-sized objects.

As previously discussed, Hansen (2009) and Walsh et al. (2011) showed that the key to the successful formation of Mars is to accumulate planet-forming material in a narrow region with an outer edge at 1 AU. As a consequence of limiting the protoplanetary disk to such a small annulus, the accretion and growth of an object will stop when it is scattered outside this region. The latter seems to present a pathway to the successful formation of Mars (or, Marsanalogs). In that respect, considering a local depletion in the protoplanetary disk around the Mars' location presents an alternative to the Grand Tack model for forming a Mars-analog using the Hansen-style disk truncation (Hansen, 2009) without the brief migration of Jupiter and Saturn in a gas-rich phase as used by Walsh et al., (2011). Another important aspect of this scenario is that unlike the model by Hansen (2009) which does not track the delivery of water to the terrestrial zone due to its initial adhoc set up of the protoplanetary disk, it presents a water-delivery mechanism that is also different from that of Walsh et al. (2011) who suggested that the water is delivered to around 1 AU very early during the outward migration of Jupiter and Saturn.

In this paper, we examine this scenario by simulating the formation of terrestrial planets in a disk with a local minimum in surface density as proposed by Chambers \& Cassen (2002) and Jin et al (2008). Our goal is to determine the range of the parameters for which a 
protoplanetary disk with a locally depleted region can form a Mars-sized planet around 1.5 $\mathrm{AU}$, and also produce a planetary system (fairly) consistent with the inner solar system (i.e., with Venus- and Earth-sized planets and with appropriate amount of water on Earth). We consider the local minimum to be around Mars' semimajor axis and use a non-uniform distribution of planetesimals and planetary embryos to simulate the final stage of terrestrial planet formation. Because during the evolution of the disk, the location of the local minimum may vary, we will carry out simulations for different values of the location and depth of this region.

It is important to note that according to Jin et al. (2008), the inward flow of the material from outer regions of the nebula to its inner parts due to viscosity differences might have affected the composition of the disk material prior to the accretion of terrestrial planets. For instance, volatile-rich dust grains or planetesimals could have drifted inward much earlier than the onset of terrestrial planet formation, and have played an important role in the final mass and composition of these objects. The study of the changes in the properties of the nebula due to the inward flow of material and appearance of the gap is a complicated task that has not been accounted for in any rigorous ways in current protoplanetary gas-disk models. Such a modeling is also beyond the scope of this paper. In this study, we avoid these complications by focusing solely on the last stage of the accretion of planetesimals and planetary embryos. For simplicity, we also neglect the re-accumulation of the material that was originally removed from the depleted region of the disk during the evolution of the nebula, in particular the material that might have drifted inward into the terrestrial planet region. Due to the lack of a proper model for the evolution of the nebula, an initial condition for planetesimals and planetary embryos that takes into account such a re-accretion of the drifted material would be poorly constrained.

We describe our model in section 2 and present the results of our simulations in section 3. Section 4 concludes this study by summarizing the results and discussing their implications for the formation of terrestrial planet in our solar system.

\section{The Model}

As shown by Kokubo \& Ida (1998, 2000), during the formation of terrestrial planets, the Runaway and Oligarchic growths of planetesimals and planetary embryos result in a bi-modal distribution of mass in the protoplanetary disk. Since in this study, our focus is on the late stage of terrestrial planet formation, in order to be consistent with the bi-modality of the disk, we consider a protoplanetary disk of planetesimals and planetary embryos with half of its mass from the planetesimals and the other half from planetary embryos. The disk 
extends from 0.5 AU to $4 \mathrm{AU}$. In such a bimodal disk, the planetesimals provide dynamical friction which is necessary to damp the eccentricities and inclination of the planetary embryos embedded in the disk (O'Brien et al. 2006; Morishima et al., 2008). The individual planetesimals are considered to have a mass of 0.0025 Earth-masses and distributed with a surface density profile of $r^{-3 / 2}$. These objects only interact with the planetary embryos and the giant planets, and do not see each other. The masses of the planetary embryos scale as $M \sim r^{3 / 2(2-\alpha)} \Delta^{3 / 2}$ (Kokubo \& Ida 2000; Raymond et al. 2005, 2009) where $\alpha$ is a free parameter and $\Delta$ is the number of mutual Hill radii. We consider the embryo-to-planetesimal mass-ratio to be $\sim 8$ around 1.5 AU (Raymond et al. 2009), and the disk surface density to have a radial profile of $r^{-3 / 2}$ (i.e., $\alpha=3 / 2$ ) and be given by

$$
\Sigma(r)= \begin{cases}\Sigma_{1}(r / 1 \mathrm{AU})^{-3 / 2} & ; \text { outside the depleted region, } \\ (1-\beta) \Sigma_{1}(r / 1 \mathrm{AU})^{-3 / 2} & ; \text { inside the depleted region. }\end{cases}
$$

In this equation, $\Sigma_{1}=8 \mathrm{~g} / \mathrm{cm}^{2}$ and the parameter $0<\beta \leq 1$ represents the scale of local mass-depletion. Figure 1 shows the distribution of planetary embryos and planetesimals for $\beta=50 \%$. We chose the location of the local mass-depletion according to the model presented by Jin et al. (2008). However, because as shown by these authors, this location changes in time, in order to better explore the parameter space of the system, we considered two different regions of mass-depletion; one extending from 1.1 AU to 2.1 AU denoted as the disk model A, and one extending from 1.3 AU to 2.0 AU denoted as the disk model B. Table 1 shows these regions and their corresponding parameter $\beta$. We assume that in the beginning of our simulations, Jupiter and Saturn are fully formed, and carry out simulations considering different orbital configuration of these planets.

We would like to recall that our goal is to present a model capable of forming terrestrial planets, in particular Mars, consistent with the inner planets of our solar system. Our approach is to follow the idea presented by Hansen (2009) (in which terrestrial planets are formed in a truncated disk of protoplanetary bodies), however, instead of appealing to the giant planet migration to create a truncated disk as in the Grand Tack scenario (Walsh et al. 2011), we consider the disk truncation to be a consequence of the disk's natural evolution. In that respect, the protoplanetary disk in the Hansen (2009) model can be considered as an extreme case of our disk model, extending from $0.7 \mathrm{AU}$ to $1 \mathrm{AU}$ with a depletion factor of $\beta=100 \%$ for the region beyond $1 \mathrm{AU}$. The fact that in our disk model, the region between 2 and $4 \mathrm{AU}$ is initially populated by planetesimals and planetary embryos (Table 1 ) is a distinct characteristic of our model that makes the results more realistic and, unlike Hansen's simulations, allows for the formation of the asteroid belt and the delivery of water 
to Earth from water-rich planetesimals and protoplanetary embryos (Morbidelli et al., 2000; Raymond et al., 2004).

\section{Numerical Simulations}

We performed a total of 84 simulations considering two different sets of initial orbital configurations for the giant planets. As mentioned before, we assumed that at the beginning of the simulations these planets were fully formed and that the gas disk had been fully dissipated. In the first set of simulations, we considered Jupiter and Saturn to be in their current orbits. In the second set, we assumed their orbital elements to be similar to those proposed in the Nice Model, that is, $a_{\text {Jup }}=5.45 \mathrm{AU}, a_{\text {Sat }}=8.18 \mathrm{AU}$, and both planets to be initially in circular and co-planar orbits (Tsiganis et al., 2005; Gomes et al., 2005; Morbidelli et al., 2005). Each simulation started with 700-950 planetesimals and 110-190 planetary embryos, all initially in circular orbits. The planetary embryos were spaced from one another at distances of 3-6 mutual Hill radii (Kokubo \& Ida, 2000). The initial orbital inclinations of all bodies were chosen randomly from the range of $10^{-4}$ to $10^{-3}$ degrees, and their mean anomalies were taken to be between $0^{\circ}$ and $360^{\circ}$. The arguments of periastrons and longitudes of ascending nodes of all objects were initially set to zero. For each value of the depletion parameter $\beta$ and giant planet configuration, we considered at least three different, randomly generated initial conditions for planetesimals and planetary embryos. In view of the stochastic nature of this kind of simulations, we performed additional simulations

using those parameters that produced planetary systems with features close to those of the solar system. We also used the water distribution model by Raymond et al. (2004) and considered planetary embryos and planetesimals inside 2.0 AU to be initially dry, the ones between $2 \mathrm{AU}$ and $2.5 \mathrm{AU}$ to carry $0.1 \%$ water, and the water contents of objects between $2.5 \mathrm{AU}$ and $4 \mathrm{AU}$ to be $5 \%$.

Using the hybrid integrator of the N-body integration package MERCURY (Chambers 1999), we integrated our systems for $500 \mathrm{Myr}$. The time-steps of integrations were set to 6 days. At the end of each simulation, we identified those that produced a potential Marsand/or an asteroid belt analog, and integrated them for another 500 Myr.

We would like to emphasize that this time of integration is considerably larger than the time of the simulations of terrestrial planet formation that is usually found in the literature. In general, most of the simulations of the late stage of terrestrial planet formation are carried out for 100 to 200 Myr (e.g., Chambers 2001, Raymond et al. 2004, 2006,2009, O'Brien et al. 2006, Hansen 2009, Walsh et al. 2011). We decided to carry out simulations for longer times because we noticed that after 200 Myr of integration, several of our simulations produced 
small bodies (including two or three potential Mars-analogs) in orbits interior to 2 AU. To examine whether these systems would be stable, we extended the integrations to longer times.

Except for planetesimal-planetesimal interactions, we allowed all objects to interact with one another and collide. It has been shown by Kokubo \& Genda (2010) that the assumption of inelastic collisions in simulations of terrestrial planet formation has no important effect on the mass and orbital assembly of the final planets (if a collision results in fragmentation, the remaining material will be accreted in subsequent collisions). In a recent study, Chambers (2013) also found that in the simulations of terrestrial planet formation where fragmentation is considered, the final planetary systems are broadly similar to those of the simulations in which fragmentation is neglected. We therefore considered collisions to be perfectly inelastic, resulting in the complete merging of the two colliding bodies while conserving linear momentum.

\section{Results}

In analyzing our results, we define an Earth-analog as a planet that is formed in the region of 0.75-1.25 AU and has a mass equal to or near that of Earth. Similarly, a Marsanalog is defined as a planet with a mass equal to or near that of Mars $\left(\sim 0.3 M_{\oplus}\right)$, formed between 1.25 AU and 2.0 AU. We also define a terrestrial planet as an object larger than 0.025-Earth masses ( 0.5 Mercury-mass) with a semimajor axis smaller than 2 AU.

As mentioned in section 3, we carried out simulations for two different orbital architectures of Jupiter and Saturn (their current orbits as well as when they are initially in circular orbits as in the Nice model), and for different values of the scale and location of the local mass depletion. In the following, we present the results of each of these simulations, and compare the results with the current state of the inner planets of the solar system.

\subsection{Jupiter and Saturn in their current orbits}

The first analysis of our results in this case indicates that in close to $50 \%$ of our simulations, the final planetary system includes at least one object with a mass smaller than half of the mass of Earth around 1.5 AU. Tables 2 and 3 show the statistics for this body in its final planetary system. Results in Table 2 correspond to the simulations of the disk model A (Table 1) and those in Table 3 correspond to the disk model B. The roman numbers in the first column of these tables refer to the first, second, and third set of initial conditions 
in the simulations for a given mass-depletion coefficient.

Figure 2 shows the snapshots of the dynamical evolution of a sample system corresponding to a simulation using the disk model A (Table 1) and for a depletion scale of $75 \%$ (simulation A-75\%-II in Table 2). As shown by the bottom-right panel, integrations resulted in the formation of four planets in the region between $0.5 \mathrm{AU}$ and $1.5 \mathrm{AU}$ with the masses of (from left to right) $0.21,0.95,0.55$ and $0.08 M_{\oplus}$, respectively. The color of each body represents its water-mass fraction. The least massive planet at $1.5 \mathrm{AU}$ is an interesting low-mass object that has reached $90 \%$ of its mass in less than $2.5 \mathrm{Myr}$. The relatively fast formation of this body is in agreement with the timescale of the formation of Mars as suggested by Nimmo \& Kleine (2007) and Dauphas \& Pourmand (2011). As argued in the latter article, isotopic analyses require that Mars to have been accreted rapidly and reached approximately half of its present size in only $1.8_{-1.0}^{+0.9} \mathrm{Myr}$.

Figure 3 shows the results of a simulation for similar mass-depletion scale and initial orbital configuration of Jupiter and Saturn as in Figure 2, but using the disk model B (simulation B-75\%-III in Table 3). As shown by the bottom-right panel, in this simulation, three terrestrial planets are formed with masses equal to $0.7,0.98$, and $0.06 M_{\oplus}$. Given the masses and distances of these planets to the central star, this system shows a close similarity to the Venus-Earth-Mars configuration in our solar system. However, unlike the results of the simulation of Figure 2, the planet around 1.5 AU in Figure 3 accreted more slowly and took longer than 80 Myr to reach $90 \%$ of its current mass.

Figure 3 also demonstrates a case for which the extension of integrations to 1 Gyr was necessary. In this simulation, as shown by the panel corresponding to $200 \mathrm{Myr}$, a moderate number of small bodies, with two potential Mars-analogs, were formed with orbits between 1.0 AU and 2.0 AU. The extension of the integration to over $500 \mathrm{Myr}$ revealed that only one of these bodies was stable, and the other one was ejected from the system after a closeencounter with the proto-Earth at $\sim 530 \mathrm{Myr}$. This is a typical outcome that was obtained in several of our simulations. In systems with high scales of depletion, in particular, the depleted region of the disk is initially populated by a large number of small bodies. If these objects are sufficiently small, their mutual gravitational interactions will be weak, and as long as no external perturbation affects their dynamics, their orbits will stay stable for long times. However, if the sizes of these objects allow for their actual interactions to be strong, they may scatter each other, in which case the orbits of some of these bodies may become unstable.

One characteristic of the two Mars-analogs shown in Figures 2 and 3 is their moderate orbital inclinations relative to the orbit of the largest body in their systems. We note that we compute orbital inclinations relative to the orbit of the largest planet in the inner part of 
the system in order to be able to compare our results with those in Hansen (2009). Unlike the orbit of Mars that has an inclination of $\sim 2^{\circ}$ with respect to the orbital plane of Earth, the two Mars-analog planets in Figures 2 and 3 have inclinations in the range of $2^{\circ}-13^{\circ}$ with respect to the plane of the orbit of the largest planet in the region between $0.5 \mathrm{AU}$ and 1.5 AU. Our simulations show that moderate inclinations appear mainly for planets smaller than $0.1 M_{\oplus}$, probably due to the weak action of the dynamical friction on these bodies. This enhanced inclination has also been reported by Hansen (2009) in his simulations of the formation of terrestrial planets where this author showed that Mars analogs are formed with similar inclinations in almost $50 \%$ of the cases. In our simulations, the moderate inclinations of planetesimals and planetary embryos between 1.5 AU and 2.0 AU can be attributed to the effect of the $\nu_{16}$ resonance. This resonance, as well as the secular resonance $\nu_{6}$ occur

when the periods of the nodal and apsidal precessions of the orbit of a small body become equal to those of Saturn. As shown in Figure 4, the $\nu_{16}$ resonance significantly increases the inclinations of objects within the first $10 \mathrm{Myr}$.

In addition to the $\nu_{16}$ resonance, Figures 2 and 3 also show the strong effects of the meanmotion resonances with Jupiter as well as the effect of the $\nu_{6}$ secular resonance with Saturn (see the upper right panel in each figure). As shown here, these resonances increase the eccentricities of objects in their vicinities causing many of these bodies to be either scattered out of the system, or collide with the Sun, Jupiter, or Saturn. (Gladman et al., 1997, Levison \& Agnor 2003, Raymond et al. 2006, Haghighipour et al. 2013). Although these processes remove a significant amount of water-carrying objects from the protoplanetary disk (see middle-right panels of Figures 2 and 3), simulations indicate that despite a depletion scale of $75 \%$, terrestrial planets can still form around $1 \mathrm{AU}$ and carry a significant amount of water. As a point of comparison, The lower limit of the amount of the Earth's water is $5 \times 10^{-4}$ of its water-mass fraction (Raymond et al., 2004). The planets around 1 AU in Figures 2 and 3 carry higher amounts.

\subsubsection{The effect of the scale of mass-depletion}

As expected, the growth of planetary embryos in the mass-depleted region is proportional to the amount of mass that is available in that region. In other words, it depends on the depletion factor $\beta$. For large values of $\beta$ (e.g., $75 \%$ which corresponds to a total initial mass of $\sim 0.25 M_{\oplus}$ in the region of 1.3-2.0 AU in disk model B), the small amount of mass in the depleted region combined with the effect of $\nu_{6}$ and $\nu_{16}$ resonances has a negative impact on the rapid formation of Mars-analogs during the first few Myr of integration. The increase in the orbital inclinations causes planetesimals and planetary embryos to be in dif- 
ferent orbital planes, decreasing the rate of their collisions and consequently the efficiency of their growth. The orbital excitation of these bodies (as a result of both increasing their eccentricities and inclinations), causes many of these objects to be scattered out of the system devoiding the region from material necessary to form small planets and Mars-analogs (Haghighipour et al., 2013). For smaller values of $\beta$ where the mass of the depleted region is larger (e.g., 20\% which corresponds to a total initial mass of $\sim 0.82 M_{\oplus}$ in the region of 1.3-2.0 AU in disk model B), the collision and growth of planetary embryos results in the formation of larger objects. For instance, simulations with $\beta=20 \%$ routinely produced planets around 1.5 AU up to 5 times larger than Mars. This result is consistent with those in Raymond et al. (2009).

The results of our simulations also show that embryos that originated in the non-depleted region of the disk and were scattered into the depleted region seem to have a better chance of growing to the Mars size and maintaining long-term stability. These objects are considerably larger than the planetary embryos native to the depleted region and less responsive to the perturbing effects of $\nu_{6}$ and $\nu_{16}$ resonances. The latter is primarily due to the effect of the dynamical friction which strongly damps the eccentricities and inclinations of these objects. For instance, both Mars-analogs of Figures 2 and 3 were formed at larger distances $(\sim 2.7$ $\mathrm{AU}$ and 3.1 AU, respectively) and were scattered into the region of $\sim 1.5 \mathrm{AU}$ as a result of successive close-encounters with other planetary embryos and the effect of resonances (Figure 5).

The formation of Mars-analogs outside the region around 1.5 AU and their subsequent scattering into this region has also been observed in the simulations by Hansen (2009) and Walsh et al. (2011). As shown by these authors, in those simulations in which a Mars-analog was formed, this object was accreted in the region around $1 \mathrm{AU}$ and was scattered to the vicinity of the Mars' orbit at 1.5 AU. In that region, the object maintained an isolated orbit, and as a result, keeping a low mass.

In our simulations, Mars-analogs are formed both in the region interior to $1.5 \mathrm{AU}$ (e.g., around $1 \mathrm{AU}$ ) where they are scattered outward, and exterior to this region at 2.0-2.5 AU, where their interactions with other embryos and resonances causes them to scatter inward. This is a significant result in the sense that it is consistent with the formation of Mars as a stranded planetary embryo, and implies that depending on the original region of the accretion of this object, its internal composition will be different. Those Mars-analogs that are scattered inward from the regions beyond 2.0-2.5 AU appear in 25\% of our simulations and in general carry more water than those scattered outward from the inner part of the protoplanetary disk (the remaining 75\%). Depending on the regions of their origin, these objects will also have different D/H ratios than that of Mars (Drouart et al. 1999, Morbidelli 
et al. 2000). At the moment, the primordial value of the D/H ratio of Mars' water is unknown (Lunine et al., 2003), however, evidence from Martian meteorites suggests that the initial $\mathrm{D} / \mathrm{H}$ value for Martian interior water is consistent with a chondritic D/H composition (Usui et al., 2012). The formation of Mars as an inward-scattered planetary embryo can also explain the higher D/H ratio for Mars's water relative to that of Earth (Lunine et al., 2004).

The scale of depletion has also an important effect on the radial mixing of the bodies and the material-content of the final planets. Radial mixing is driven by the mutual interactions between protoplanetary bodies as well as the perturbations due to giant planets. Embryoembryo and embryo-planetesimal interactions increase the eccentricity of these objects and scatter them into other regions of the disk. Mean-motion and secular resonances with Jupiter and Saturn (e.g., in the range of $1.8 \mathrm{AU}$ to $3.7 \mathrm{AU}$ when these planets are in their current orbits) also play important roles, in particular in the delivery of water-carrying asteroid to terrestrial planets (Raymond et al., 2004). Our simulations indicate that when the depletion scale is low, the embryos inside the depleted region are initially larger and as a result, have stronger interactions with their neighboring embryos and planetesimals. In this case, radial mixing is more efficient and the material-contents of the final planets show greater diversity in their origin.

Figures 6 and 7 show samples of the results. Figure 6 corresponds to the simulations using the disk model A, and Figure 7 shows the results of the simulations using the disk model B. The size of each body in these figures corresponds to its relative physical size scaled as $M^{1 / 3}$. However, it is not to scale on the $x$-axis. The color of each object represents the relative contribution of material from different parts of the disk. The eccentricity of each planet is represented by a horizontal line corresponding to its variation in heliocentric distance over the semimajor axis. As shown in these figures, in systems with low massdepletion scales, the water-mass fraction of the final bodies are larger indicating that in such systems, the final terrestrial planets tend to carry more water (left plots in Figure 8). As shown in Figures 6-8, when the scale of depletion is 100\%, terrestrial planets are formed interior to $\sim 1.3 \mathrm{AU}$ with little or no amount of water. However, a scale of depletion of $75 \%$

or smaller produces Earth-analogs with mean water-mass fractions consistent with the value expected for Earth (Table 3).

\subsubsection{The effect of the location of the mass-depletion}

As mentioned earlier, we carried out simulations for different locations of the disk local mass-depletion. Results indicated that the position of the inner edge of this region plays a significant role in the mass and orbital assembly of the final terrestrial planets. A comparison 
between the results shown in Figures 6 and 7 indicates that for instance, in simulations where the depleted region started at 1.1 AU (Figure 6), the planets formed around 1 AU were consistently smaller than Earth. However, in simulations of Figure 7, where the inner edge of the depleted region is at $1.3 \mathrm{AU}$, these planets are relatively larger. This can be explained noting that for similar mass-depletion factors, in the simulations of Figure 6 (using the disk model A), the amount of the mass available for accretion by embryos around $1.0 \mathrm{AU}$ is smaller and as a result, the final planets have lower masses compared to those in Figure 7 where simulations were run for the disk model B.

Figure 9 shows the mean planet mass for all our simulations. The top panel in this figure includes all the planets in the system whereas in the bottom panel only those formed in the region between $0.75 \mathrm{AU}$ and $1.25 \mathrm{AU}$ have been included. As shown by this figure, the disk model $\mathrm{B}$ is more efficient in forming Earth-sized planets. It is interesting to note that in contrast to our model, Hansen (2009) and Walsh et al. (2011) used a narrower disk that was truncated at $1 \mathrm{AU}$ and were still able to form Earth-sized planets around that region. However, in their disk models, the initial amount of mass between $0.7 \mathrm{AU}$ and $1 \mathrm{AU}$ is $2 \mathrm{M}_{\oplus}$ which is almost twice higher than the amount of mass between $0.5 \mathrm{AU}$ and $1 \mathrm{AU}$ in our protoplanetary disks.

The outer edge of the depleted region (at 2.0 AU or 2.1 AU, see Table 1), did not seem to have a strong influence on the mass and orbital architecture of the final terrestrial planets. However, this boundary may play an important role in the efficiency of the delivery of water-carrying objects to the Earth's accretion zone. A mass-depleted region extending to distances well beyond $2 \mathrm{AU}$ (e.g., along all the asteroid belt) can hinder the delivery of water to Earth. We carried out simulations considering a depleted region extending from 1.5 AU to 2.5 AU and assuming Jupiter and Saturn initially in their current orbits. Results showed that for depletion scales of $50 \%$ and $75 \%$, simulations produced Earth-analogs that were mainly dry.

\subsection{Jupiter and Saturn in Circular Orbits}

To examine the role that the current orbital eccentricities of Jupiter and Saturn play in the formation of a Mars-analog and the final assembly of terrestrial planets, we also carried out simulations assuming Jupiter and Saturn to be initially in circular orbits. We considered the initial orbital elements of these planets to be identical to those in the Nice model (Tsiganis et al. 2005) and carried out simulations for 1 Gyr. Figure 10 shows the results of one of such simulations. As shown here, four planets are formed with semimajor axes smaller than 2 AU. Among these planets is an object similar to Mars with a mass 
of $\sim 0.24 M_{\oplus}$ at $\sim 1.35 \mathrm{AU}$. Although the formation of this planet can be considered as a success in forming Mars-analogs, there is another terrestrial planet in this system with a mass of $0.7 M_{\oplus}$ in a stable orbit at $1.7 \mathrm{AU}$ which has made its final planetary configuration inconsistent with the current architecture of the solar system.

The lack of consistency between the final planetary orbits and the current orbits of terrestrial planets was observed in the results of all simulations in which Jupiter and Saturn were initially in circular orbits. Figure 11 shows the mass and semimajor axes of the final bodies. As shown here, in general, results are very different from those of the previous simulations where Jupiter and Saturn were initially in their current orbits (Figures 2, 3 and 15). Unlike those simulations, when giant planets were assumed to be in circular orbits, the final planetary systems did not contain a Mars-sized planet around 1.5 AU. This was irrespective of the scale of the mass-depletion as well as the disk model. Results show that in all these simulations, only one planet with a mass smaller than $0.3 M_{\oplus}$ was formed around 1.5 AU (Figure 11).

The lack of success in forming Mars-sized planets around 1.5 AU in the simulations in which Jupiter and Saturn were initially in circular orbits is in contrasts with the results of the simulations by Walsh et al. (2011) who also considered Jupiter and Saturn to be in circular orbits and were still able to produce Mars-analogs. The reason for this discrepancy can be found in the mass-distribution in the disk models used by these authors. At the end of the inward migration of Jupiter and Saturn, these authors set the total initial mass available for accretion in the terrestrial zone to $\sim 2 M_{\oplus}$. They also considered that the region of the accretion of terrestrial planets is confined to a narrower region from $\sim 0.7 \mathrm{AU}$ to 1 AU. After the inward-then-outward migration of Jupiter and Saturn, the total mass of the remaining planetesimals and protoplanetary embryos orbiting from 1.5 AU to 4 AU, in their model becomes very small. As a result, these objects do not contribute significantly to the growth of the planetary bodies in the terrestrial zone. In our simulations, however there always exist a significant amount of mass $\left(\sim 1.5 M_{\oplus}\right)$ beyond 2.5 AU. When the orbits of Jupiter and Saturn are considered to be circular, their interactions with the protoplanetary disk are minimal and as a result, the disk maintains a large portion of its original mass. Consistent with previous studies in which Jupiter and Saturn were assumed to be in circular orbits (e.g., Wetherill 1996; Raymond et al. 2005, Kokubo et al. 2006), a more massive disk in these simulations produces more massive objects. Figure 12 shows the mean mass of the planets formed in our simulations considering different giant planet configurations.

The smaller perturbations of the giant planets on the protoplanetary disk in simulations in which these planets are considered to be in circular orbits also affects the water contents of the terrestrial planets. In these simulations, the radial mixing of the protoplanetary bodies 
is more effective and results in forming planets with higher contents of water (Figure 8, see also Raymond et al. 2009). That is because more water-carrying objects from the outer part of the protoplanetary disk maintain their orbits for longer times. A comparison between the result of the simulations shown in Figures 3 and 10, which correspond to the same disk model, indicates that in the simulations where Jupiter and Saturn are in their current orbits, the protoplanetary disk loses a large portion of its water-carrying objects as a result of the stronger interactions of these giant planets with the planetesimals and planetary embryos. We expect comparable results to be obtained using an updated version of the Nice model (Levison et al., 2011) as well.

\subsubsection{The effect of the scale and location of the mass-depletion}

When Jupiter and Saturn are considered in circular orbits, our simulations do not show a clear correlation between the results and the different values of the scale and location of the mass-depletion (Figures 11-13). As discussed above, when the giant planets are in circular orbits, the protoplanetary disk is perturbed only weakly, and it maintains a higher fraction of its original mass for longer times. The effect of the scale of the mass depletion in this case vanishes probably because a significant part of the material in the neighborhood of the depleted region enters in the depleted area during the evolution of the system. This material comes mainly from the outer part of the protoplanetary disk as the gravitational effects of Jupiter and Saturn are weak, and only remove a small fraction of the disk bodies.

\section{Comparison with solar system and other simulations}

To compare the results of our simulations with the current orbital architecture of terrestrial planets in our solar system and with the results of simulations by other authors, we calculated the radial mass concentration statistics (RMC) and angular momentum deficit (AMD) of the final planetary systems of our simulations. The value of RMC varies with the semimajor axes of planets and is given by (Chambers 1998, 2001; Raymond et al. 2009)

$$
\mathrm{RMC}=\operatorname{Max}\left(\frac{\sum_{j=1}^{N} m_{j}}{\sum_{j=1}^{N} m_{j}\left[\log _{10}\left(a / a_{j}\right)\right]^{2}}\right) .
$$

In this equation, $m_{j}$ and $a_{j}$ are the mass and semimajor axis of planet $j$, and $N$ is the number of final bodies. The AMD of a system represents a measure of the deviation of the actual orbital angular momentum of the planets from the total angular momentum of the 
system, had the planets been in circular and co-planar orbits. Following Laskar (1997), we use equation (3) to calculate this value,

$$
\mathrm{AMD}=\frac{\sum_{j=1}^{N}\left[m_{j} \sqrt{a_{j}}\left(1-\cos i_{j} \sqrt{1-e_{j}^{2}}\right)\right]}{\sum_{j=1}^{N} m_{j} \sqrt{a_{j}}} .
$$

In this equation, $e_{j}$ is the orbital eccentricity of planet $j$, and $i_{j}$ is its inclination with respect to an invariant plane.

Figures 6 and 7 show the values of the RMC and AMD of their corresponding planetary systems. As a point of comparison, the inner planets of the solar system are also shown. We recall that in these simulations, Jupiter and Saturn were initially in their current orbits. We would also like to note that, similar to the planet V proposed by Chambers (2007), some of our simulations produced small planets (mainly unaccreted embryos) past 2 AU. However, we did not take these planets into account when calculating RMC and AMD values.

Figures 13 and 14 show the mean values of the RMC and AMD of our simulations for different scales of the mass-depletion, disk models, and giant planet configuration. The circular solid points in these figures represent the means of the RMC and AMD calculated from a set of planetary systems produced by at least three different simulations with the same mass-depletion scale. The vertical bars on each point represent the lower and upper values of the RMC and AMD in the sample over which their means were calculated. The values of the RMC and AMD of Mercury, Venus, Earth and Mars (hereafter MVEM) are also shown in these figures. A comparison between these values with the values of the RMC and AMD of the sample results shown in Figures 6 and 7, and the mean values shown in Figures 13 and 14 indicates that the mean RMC of our planetary systems are significantly smaller than that of MVEM. The higher value of the RMC of MVEM is mainly due the orbital proximity of these planets and the comparable masses of Venus and Earth. The lower values of the mean RMC in our results indicate that our simulations form planets in more widely spread orbits than the separation of the orbits of MVEM.

Our results also indicate that, except for a few planetary systems, the mean AMD of our simulations is, in general, slightly higher than the AMD of MVEM. This is mainly due to the initial distribution of the total mass of the disk among planetesimals and planetary embryos which can be adjusted to result in systems with lower AMDs. As shown by O'Brien el al. (2006), it is possible to obtain lower AMDs by increasing the initial value of the planetesimal/embryo mass-ratio while keeping similar distribution of disk mass between the embryo and planetesimal populations (e.g., assigning $50 \%$ of the disk mass to planetesimals and $50 \%$ to the planetary embryos). The higher values of the planetesimal/embryo mass- 
ratio will enhance the dynamical friction which will then be more effective in reducing the value of AMD. The dynamical friction can also be enhanced if a residual population of very small objects still exist in the inner solar system after the terrestrial planet formation is completed (Schlichting et al., 2012).

To compare our results with previous studies, we first consider the simulations of the formation of terrestrial planets by Raymond et al. (2009). A comparison between the values of the RMC of our systems and those from these authors indicates that only when in their simulations, Jupiter and Saturn are initially in eccentric orbits $(e \sim 0.1)$, the values of their RMC are similar to those in our best models in which a Mars-analog is formed. The reason is that in their simulations, the eccentric orbits of Jupiter and Saturn cause these planets to have strong interactions with the objects in the outer part of the disk, removing the majority of them from the system in a short time and creating an edge for the disk at $\sim 2$ AU (few million years, Raymond et al. 2009). As the material is removed from the disk, the $\nu_{6}$ secular resonance shifts interior to 2.0 AU (Gomes, 1997; Haghighipour et al., 2013) and continues to remove material from the inner part of the disk. This causes the disk to develop a local mass-depleted region similar to the one considered in our disk models. We note that although the radial mass concentrations in all our simulations are smaller than that of the terrestrial planets, in general, they are higher than those in most of the simulations by Raymond et. al (2009).

A comparison of our results with those in the simulations by Hansen (2009) and Walsh et al. (2011) shows that the values of their RMC are higher than the average value found in our results. The reason can be attributed to the extent of the protoplanetary disks in those studies. Both in simulations by Hansen (2009) and Walsh et al. (2011), the total mass necessary for the accretion of terrestrial planets was distributed over a small region between 0.7 AU to 1 AU. Such a narrow distribution will naturally result in the formation of planetary systems with high values of RMC. Recall that in our simulations, similar to the most of the previous studies of terrestrial planet formation in the solar system (e.g., Raymond et al. 2009), the protoplanetary disk extends from 0.5 AU to 4 AU. As shown by our results, it is possible to form planetary systems with higher values of RMC in such disks when Jupiter and Saturn are considered in their current orbits by using a large mass-depletion scale (Figure $13)$.

The results of our simulations indicated that Mars-sized planets have a better chance of forming around 1.5 AU in systems where Jupiter and Saturn are initially in their current orbits and when the mass-depletion factor has a moderately large value (50\% - 75\%). For the purpose of extending our analysis, we identified this subset of our simulations, and following Raymond et al. (2009) and recalling our definitions of Mars- and Earth-analogs in Section 
4, we evaluated their success, quantitatively, in producing

- a Mars-analog with a mass smaller than $0.3 M_{\oplus}$ in less than $10 \mathrm{Myr}$ (with half-accretion in less than $2.7 \mathrm{Myr}$ ),

- an Earth-analog with a mass larger than $0.7 M_{\oplus}$ and a water-mass fraction larger than $5 \times 10^{-4}$ in $30-150 \mathrm{Myr}$,

- a system of terrestrial planets with AMD $<0.0036$ (twice the MVEM value), and

- no stranded embryos in the asteroid belt with masses bigger than $0.05 M_{\oplus}$.

The results are presented in Tables 4 and 5 . As shown in these tables, different combinations of the scale and location of the disk local mass-depletion can result in systems that in many occasions partially, and in some specific cases almost entirely satisfy the above-mentioned requirements. When the scale of the local mass-depletion in the disk is moderately large (e.g., $\beta=50 \%-75 \%$ ), the final systems are more successful in meeting the above mentioned criteria, especially when the depleted region is considered to be between 1.3 AU and $2 \mathrm{AU}$ (Table 5).

\subsection{Formation of asteroid belt analogs}

One important feature of our solar system that imposes strong constraints on the models of terrestrial planet formation and solar system dynamics is the asteroid belt. Any model for the formation of the inner planets has to also be able to account for the existence of small bodies between 2.1 AU and 3.2 AU, and their orbital architecture. In our simulations, several systems showed signs of asteroid belt analogs. For instance, in the simulations of Figure 3, three planetesimals remained stable in the region of $2 \mathrm{AU}$ to $3.5 \mathrm{AU}$, for the duration of the integration $(1 \mathrm{Gyr})$. This simulation also produced an Earth-analog $\left(\sim 1 M_{\oplus}\right)$ around $1 \mathrm{AU}$ in less than $150 \mathrm{Myr}$, which is consistent with the timescale of the formation of the EarthMoon system (Jacobsen 2005; Touboul et al 2007). The innermost planet in this system is a Venus-analog.

The final number of planetesimals in the region between $2 \mathrm{AU}$ and $3.5 \mathrm{AU}$, i.e. their long-term (> $100 \mathrm{Myr}$ ) stability, depends on the interactions between these objects and their

neighboring planetary embryos. The latter itself depends on the interaction of giant planets with the protoplanetary disk. Figure 15 shows the final distribution of surviving bodies in those simulations that had full or relative success in producing Mars-analogs around 1.5 AU 
(see also Table 5). As shown here, many embryos with long-term stable orbits are formed in the asteroid belt. Quantitatively, in 14 simulations out of 18 shown in Figure 15, either no embryo was left in the asteroid belt region, or if there was any embryo, its mass was smaller than $0.05 M_{\oplus}$. These results agree with the findings of Raymond et al. (2009) who showed that in systems where giant planets are in slightly excited orbits, some embryos may maintain their orbits in the asteroid belt region for $200 \mathrm{Myr}$ of integration. These authors also showed that it is improbable that during the formation of terrestrial planets, a Marssized embryo could have been stranded in the asteroid belt and maintained its orbit for 100 Myr (Raymond et al., 2009; Brasser et al., 2011). Such an embryo would have disturbed the orbits of other bodies in its vicinity, creating a gap in the protoplanetary disk that is not observed in the present day asteroid belt1. Our results also agree with this finding. In regard to the latter, we also analyzed the results of our simulations where Jupiter and Saturn were initially in circular orbits. We found that planetary embryos that survived in the asteroid belt were, in general, much larger than Mars, reaching to a mass equal to almost half of the mass of Earth (see Figure 11).

Figure 15 also shows that in the simulations in which the depletion scale is $50 \%$, the orbital elements of the population of asteroids in the region of the asteroid belt are closely similar to those of the real population of the main belt. In the simulations where the depletion scale is $75 \%$, on the other hand, asteroids were produced mainly between $2.8 \mathrm{AU}$ and $3.2 \mathrm{AU}$, with a few bodies also between 2.2 AU and 2.8 AU. We would like to note that because these simulations are very time-consuming, we adopted a moderate resolution. Higher resolution simulations may produce results that may show better agreement with the structure of the asteroid belt.

An interesting result depicted by the top panels of Figure 15 is the appearance of a dualmass population among the surviving planetesimals in the asteroid belt. One population is at 0.0025 Earth-masses outside the depleted region in both panels. The second population is around 0.00125 Earth-masses in the left panel and 0.000625 Earth-masses in the right panel. The appearance of such a dual-mass population is due to the initial assumption of the existence of a local depletion in the disk. The small objects in the asteroid belt are primarily native to the depleted area whereas the larger ones originated outside of the depletion. The native objects are also small in number (only 4 in each of the 9 simulations shown in Figure 15). The small mass and number of the native planetesimals is due to the effect of resonances which causes many of these bodies to be scattered out of the system. As a result, the asteroid belt analogs that are formed in our simulations do not carry a

\footnotetext{
${ }^{1}$ Note that this gap is different from those that are due to mean-motion resonances with giant planets.
} 
mass-gradient distribution.

The small number of the final surviving planetesimals in the region of the asteroid belt in our simulations also points to a strong clearing process during which many objects were scattered out of the system. In several of our simulations, the initial number of planetesimals in the asteroid belt was over 400. However, on average no more than 3 planetesimals survived in the end of the simulations (e.g. Figure 2 and 3). This value corresponds to a very small fraction of the initial number of planetesimals in that region which is in agreement with previous studies indicating that a vast majority of the mass of the asteroid belt was removed during the evolution of the solar system and the formation of terrestrial planets (Petit el al. 2001)

\section{Conclusion and Discussion}

We studied the late stage of the accretion of terrestrial planets in a disk of protoplanetary bodies with a locally depleted region. Following Jin et al (2008), we considered that a depletion in the density of the protosolar nebula will result in a non-uniform formation of planetesimals and planetary embryos, and studied the effects of the scale and location of such a local depletion on the mass, water-content, and final orbital assembly of terrestrial planets. For simplicity, we neglected the reallocation/redistribution of mass from the depleted region into the inner parts of protoplanetary disk due to the early radial in-flow of material (Jin et al., 2008). This enabled us to avoid complications that would rise from considering a more complex compositional and mass-size gradient for the initial distribution of solids in our protoplanetary disk models.

As expected, the final mass and orbital assembly of the planets in our simulations were strongly affected by the initial orbital configurations of Jupiter and Saturn. The results of integrations showed a clear distinction between the outcome of simulations in which the orbits of the giant planets were considered to be initially circular and those in which Jupiter and Saturn had slightly eccentric orbits. Similar to previous studies (O'Brien et al., 2006; Raymond et al., 2009), when giant planets were in circular orbits, simulations were systematically unsuccessful in forming planetary systems with Mars-analogs at 1.5 AU. In this case, even in simulations with high scale of depletion, the effect of the local disk-depletion seemed to vanish and the terrestrial planets that formed around Mars' orbit were more massive than $0.3 M_{\oplus}$. These results suggest that if Jupiter and Saturn were initially in circular orbits, as in the Nice Model (Tsiganis et al. 2005) or a recent model by Levison et al. (2011), a disk with a local mass-depletion may be able to form a Mars-sized planet around 1.5 AU only if an additional mechanism removes material from the disk in the region of the asteroid 
belt. However, such a mechanism has to also ensure that the delivery of water-carrying planetesimals to the region of Earth accretion will stay efficient.

When Jupiter and Saturn were placed in their current orbits, the interactions of these planets with the protoplanetary disk played an important role in forming Mars-analogs. Results of our simulations indicated that in this case, a significant portion of the disk material is removed from its outer regions creating a favorable condition for Mars-analogs to form around 1.5 AU when the depletion factor has a moderately large value of $50 \%$ to $75 \%$. In these simulations, when considering the disk model B, in addition to forming a Mars-analog, our models were able to deliver sufficient amount of water to their corresponding Earthanalogs in $\sim 40 \%$ of the cases (Table 5 ). The Mars-analogs in these simulations formed as an embryo that was scattered from the non-depleted region (either the inner or the outer part) into the depleted area. Simulations showed that a depleted region around 1.5 AU with a high scale of depletion increases the stability of this planet, although caution has to be taken since larger values of the mass-depletion will have negative impact on the radial mixing of planetesimals and planetary embryos, and can deprive Earth from having sufficient amount of water. Embryos scattered from the other parts of the disk to the depleted region are substantially more massive than the native planetary embryos, and as a result will have a higher chance of being stable. These embryos also tend to grow slowly since not much material will be available in the depleted area to accommodate their collisional growth.

The results of our simulations also showed that as expected, the final semimajor axes and eccentricities of Jupiter and Saturn were slightly different from their initial values. Such changes in the orbital elements of the giant planets have also been reported by Chambers \& Wetherill (2001), and Raymond et al. (2004), and are the result of the interaction of these bodies with planetesimals and planetary embryos, and the subsequent decrease in the mass of the protoplanetary disk due to the scattering and ejection of these objects from the system. In this study, at the end of the simulations in which Jupiter and Saturn were initially in their current orbits, the semimajor axis and eccentricity of Jupiter decreased by an average of $0.045 \mathrm{AU}$ and 0.035, respectively. However, no significant changes occurred to the semimajor axis of Saturn. In most case, the final semimajor axis of this planet was within a range of $\pm 0.03 \mathrm{AU}$ from its initial value. The eccentricity of Saturn decreased by 0.035 .

The above-mentioned damping of the final eccentricities of the giant planets suggests that as the formation of terrestrial planets approaches its final stage (or after these planets are fully formed), a mechanism has to exist to ensure that the orbits of Jupiter and Saturn will have eccentricities similar to their current values. One approach is to consider the initial orbital eccentricities of these planets, at the beginning of each simulation, to be large. 
Raymond et al. (2009) considered, for instance, an initial value of 0.1 for the eccentricities of both Jupiter and Saturn, and showed that the final eccentricities of these planets will be close to their current values (0.05). Whether such large orbital eccentricities are possible is, of course, a matter of debate. As shown by Lega et al. (2013), early dynamical instabilities between Jupiter and Saturn that may occur at the end of the lifetime of the gas disk could increase the orbital eccentricities of these bodies. However, Raymond et al. (2009) have presented a list of arguments as to why Jupiter and Saturn could not have evolved into such a dynamical state. Results of our study suggest that modest values of orbital eccentricities for Jupiter and Saturn are required to ensure the efficient formation of Mars-analogs. Given the subsequent damping of the eccentricities of these planets, our model also requires a latestage dynamical instability between Jupiter and Saturn, such as that proposed by Tsiganis et al., (2005) to raise the orbital eccentricities of these planets to their current values.

At the beginning of each simulation, to create a low-density region, we scaled down the masses of planetesimals and planetary embryos inside the depleted area. However, depending on the scale of the mass-depletion, the efficiency of the growth of planetary embryos from planetesimals (Kokubo \& Ida, 2000) inside the depleted area may be so low that during the Runaway and Oligarch phases, either no planetary embryo is formed, or they may not grow to large sizes. This implies that even if we had not scaled down the masses of the objects (e.g. this region was initially populated only by planetesimals), the results would not have changed and Mars-analogs would have formed in the same fashion as in our simulations. As mentioned before, Mars-sized objects originated from the regions outside the mass-depleted area and because they had more mass to accrete, they were larger than the native bodies. Once inside the deplete area, these objects had a better chance of growing to the Mars' size because they dominated this region gravitationally and accreted (or scattered) smaller, native bodies more efficiently.

The results of the simulations that were successful in forming Mars-analogs around 1.5 AU also show that unlike simulations with low or no local mass-depletion (e.g., Chambers 2001; Raymond et al. 2009), a scale of depletion of $50 \%$ to $75 \%$ is crucial for building planetary systems with stronger radial mass concentrations. Although our simulations did not produce RMC values as high as that of the current terrestrial planets, their results point to potential pathways for improving models of terrestrial planet formation using a local mass-depletion. The correlation between the scale of mass-depletion and the RMC value also indicates that the strong radial mass concentration of terrestrial planets and the low mass of Mars are two characteristics of the solar system that are deeply connected.

Despite the success of our model in forming planets similar to Mars and ensuring the delivery of sufficient amount of water to Earth, none of our simulations was able to reproduce 
all the features of the inner solar system. The hardest constraints to satisfy was the fast formation of Mars. As suggested by Nimmo \& Kleine (2007) and Dauphas \& Pourmand (2011), the measurements of the Hf/W ratio in the Martian mantle point to a timescale of 0-10 million years for the formation of the core of Mars, and a time of slightly less than 2 million years during which Mars reaches to $50 \%$ of its current mass. Although the formation of Mars-analogs around the current orbit of Mars was successful in many of our simulations (Table 4 and 5), the time during which these objects grew to $50 \%$ of their masses was in general (in more than $92 \%$ of the simulations of Table 4, and in more than $95 \%$ in the simulations of Table 5) much longer than 2 million years.

It is important to emphasize that this longer time of Mars accretion should not be considered a weakness for our model. The 0-10 million years timescale for the growth of the core of Mars, as suggested by Nimmo \& Kleine (2007), is based on the analysis of a limited number of shergottite-nakhlite-chassignite (SNC) meteorites that are assumed to have formed the bulk of Mars mantle. Also, the time of the growth of Mars to $50 \%$ of its current mass as suggested by Dauphas \& Pourmand (2011) is based on the assumption that the mass-evolution of planetary embryos during the oligarchic state can be modeled by a simple analytic solution if a uniform size of planetesimals is adopted (Chambers, 2006). However, using N-body simulations, Morishima et al. (2013) have shown that such an analytic approximation for the mass-evolution of planetary embryos does not agree with the final masses of these objects at the late stage of the oligarchic growth. These authors also showed that for a disk with a minimum mass solar nebula, the timescale for the formation of Mars maybe much longer than those derived from the Hf-W chronology - a result that is consistent with our finding as well.

When comparing the time of the formation of Mars obtained from numerical simulations such as those presented here, with those obtained from cosmochemical studies, another important factor that needs to be taken into consideration is that unlike analytical approximations of the mass-evolution of embryos, numerical simulations include the effects of Jupiter and Saturn that are assumed to have been fully formed in the beginning of simulations. This is probably 1-3 Myr after the formation of the first solids (Raymond et al. 2009), the calcium-aluminum inclusions [CAIs, dated at 4.568 billion years ago (Bouvier et al. 2007)]. As shown by many authors [see e.g., Haghighipour \& Scott (2012) and references therein], these planets have profound effects on the distribution and growth of planetary embryos during their own formation and after they are fully formed. The perturbations of these planets cause many embryos to be scattered out of the planet-forming region - a process that will have important consequences on the final masses of terrestrial planets. The analytical treatment of the growth of embryos, however, does not take the effects of giant planets into account. 
Numerical simulations of the formation of terrestrial planets such as those presented here and by Morishima et al. (2013), consistently form Mars at timescales larger than that suggested by Nimmo \& Kleine (2007) and Dauphas \& Pourmand (2011). As argued by Morishima et al. (2013) and Kobayashi \& Dauphas (2013), this time can be reduced if Mars grew by the accretion of small fragments and pebbles, or the surface density of the protoplanetary disk around the orbit of Mars is locally enhanced. This latter scenario seems to be consistent with the assumption of a local mass-depletion in the proto-solar nebula due to the variation in gas viscosity as suggested by Jin et al (2009)2. Such a depletion may cause the material to accumulate outside the depleted area, creating regions where the surface density of the disk is locally enhanced. These density-enhanced regions may produce large embryos of the size of Mars in short timescales during the Runaway and Oligarchic growth phases (Kokubo \& Ida, 2000). In our simulations, Mars-sized bodies do in fact form in the regions interior or exterior to the local mass-depletion where the disk surface density may be locally enhanced (Jin et al., 2008). These objects are then scattered into the region of mass-depletion where they maintain a stable orbit for a long time. This implies that if Mars were a planetary embryo that grew in a density-enhanced region, it could have formed in a short timescale consistent with the finding of Dauphas \& Pourmand (2011). However, we recall that as mentioned in Section 1, we do not use such local surface density enhancements when generating the initial structure of our protoplanetary at the beginning of our simulations.

One important aspect of our simulations is the treatment of the two-body collisions. In all our simulations, we assumed that collisions were perfectly inelastic and resulted in the complete merging of the two impacting bodies. We also assumed that no water was lost during an impct (Marty \& Yokochi 2006) or hydrodynamic escapes (Matsui \& Abe 1986), and the total amount of water in the final body would be equal to the sum of the water contents of the colliding objects. In more realistic simulations, the loss of volatile materials during an impact has to be taken into account (Genda \& Abe 2005, Canup \& Pierazzo 2006).

In closing we would like to emphasize that the works of Hansen (2009) and Walsh et al. (2011) provided significant context and motivation for the development of this study. Walsh et al. (2011) combined the narrow-disk idea of Hansen (2009) with giant planet

\footnotetext{
${ }^{2}$ We would like to note that the results presented by Jin et al (2009) and their suggested mechanism for generating a local mass depletion in the disk depend highly on the choice of some poorly constrained parameters such as the rotational velocity of the cloud's core and the viscosity of the gaseous disk. In fact, regions of local mass depletion may appear during the evolution of a disk for a verity of reasons, and may have long enough lifetimes to affect the formation and growth of planetesimals, and appear as local mass depletions in the protoplanetary disk as well.
} 
migration, and developed a model for the formation of Mars and delivery of water to Earth in which most of the water delivered to terrestrial zone is from primitive asteroids that were scattered inward during the outward migration of giant planets. We, however, showed that it would be possible to form a low-mass planet around Mars' semimajor axis (and deliver sufficient amount of water to Earth) without considering drastic inward migration for Jupiter and Saturn, if the protosolar nebula did have a locally depleted region around the Mars' location. The efficiency of the formation of Mars-sized planets around 1.5 AU will be high when the giant planets are considered to be farther out than initially assumed in the Grand Tack model (e.g., close to their current locations) and in slightly eccentric orbits (e.g., close to their current values). In this model, contrary to what Jin et al. (2008) proposed, that a Mars-analog would form from the embryos native to the depleted region, our simulations show that this object is formed outside the depleted area and is scattered into this region as a result of interacting with giant planets and other planetary embryos. Also, although our model uses the same narrow-disk idea as in Hansen (2009) and Walsh et al (2011), our scenario for water-delivery to Earth is completely different. In our model, similar to previous studies such as those by Raymond et al. (2009), water comes from the asteroid belt region due to the perturbing effects of giant planets. This will possibly result in the accretion of a different fraction of primitive asteroids by the forming Earth, as well as a different time for their accretion. More precise measurements are needed to determine the fraction of the material from different parts of the solar nebula that contributed to the formation of terrestrial planets and the origin of the Earth's water. Such measurements will have profound effects on constraining models of terrestrial planet formation in our solar system and can be used to differentiate between existing scenarios.

We would like to thank the referee, Kevin Walsh, for his very constructive comments that greatly improved this manuscript. We are also thankful to Alessandro Morbidelli for his carefully reading of our paper and his helpful comments. AI and OCW would like to thank Rafael Sfair for his assistance with the computing cluster that was used to run part of these simulations. AI and OCW would also like to acknowledge financial support from the Brazilian National Research Council (CNPq), Coordenacao de Aperfeicoamento de Pessoal de Nível Superior (CAPES), and FAPESP - São Paulo State Funding Agency, proc. 2011/08171-3. AI wishes to thank the Institute for Astronomy at the University of Hawaii for their kind hospitality during the course of this project. NH acknowledges support from the NASA Astrobiology Institute under Cooperative Agreement NNA09DA77A at the Institute for Astronomy, University of Hawaii, and the Alexander von Humboldt Foundation. $\mathrm{NH}$ is also thankful to the Computational Physics group at the Institute for Astronomy and Astrophysics, University of Tübingen for their kind hospitality during the course of this 
project.

\section{REFERENCES}

Agnor, C. B., Canup, R. M., \& Levison, H. F. 1999, Icarus, 142, 219

Agnor, C. B., \& Lin, D. N. C. 2012, ApJ, 745, 143

Allègre, C. J., Manhès, G., Göupel, C. 1995, Geochim. Cosmochim. Acta, 59, 1445

Bouvier, A., Blichert-Toft, J., Moynier, F., Vervoort, J. D., \& Albarède, F. 2007, Geochim. Cosmochim. Acta, 71, 1583

Brasser, R., \& Morbidelli, A. 2011, A\&A, 535, A41

Canup, R. M. \& Pierazzo, E. 2006, LPI conference series, 37, 2146

Chambers, J. E. 1998, Earth Moon and Planets, 81, 3

Chambers, J. E. 1999, MNRAS, 304, 793

Chambers, J. E. 2001, Icarus, 152, 205

Chambers, J. E. 2007, Icarus, 189, 386

Chambers, J. 2006, Icarus, 180, 496

Chambers, J. E. 2013, Icarus, 224, 43

Chambers, J. E., \& Cassen, P. 2002, Meteoritics and Planetary Science, 37, 1523

Chambers, J. E., \& Wetherill, G. W. 1998, Icarus, 136, 304

Chambers, J. E., \& Wetherill, G. W. 2001, Meteoritics and Planetary Science, 36, 381

Chiang, E. I., \& Goldreich, P. 1997, ApJ, 490, 368

Craddock, R. A., \& Howard, A. D. 2002, Journal of Geophysical Research (Planets), 107, 5111

D’Angelo, G., \& Marzari, F. 2012, ApJ, 757, 50

Dauphas, N., \& Pourmand, A. 2011, Nature, 473, 489

Drouart, A., Dubrulle, B., Gautier, D., \& Robert, F. 1999, Icarus, 140, 129 
Eisner, J. A., Hillenbrand, L. A., White, R. J., Akeson, R. L., \& Sargent, A. I. 2005, ApJ, 623, 952

Garaud, P., \& Lin, D. N. C. 2007, ApJ, 654, 606

Genda, H. \& Abe, Y. 2005, Nature, 433, 842

Gladman, B. J., Migliorini, F., Morbidelli, A., et al. 1997, Science, 277, 197

Goldreich, P., Lithwick, Y., \& Sari, R. 2004, ApJ, 614, 497

Gomes, R. S. 1997, AJ, 114, 396

Gomes, R., Levison, H. F., Tsiganis, K., \& Morbidelli, A. 2005, Nature, 435, 466

Haghighipour N., Izidoro A., Winter O. C. 2013, submitted

Hansen, B. M. S. 2009, ApJ, 703, 1131

Horner, J., Mousis, O., \& Hersant, F. 2007, Earth Moon and Planets, 100, 43

Izidoro, A., de Souza Torres, K., Winter, O. C., \& Haghighipour, N. 2013, ApJ, 767, 54

Jacobsen, S. B. 2005, Annual Review of Earth and Planetary Sciences, 33, 531

Jin, L., Arnett, W. D., Sui, N., \& Wang, X. 2008, ApJ, 674, L105

Kobayashi, H., \& Dauphas, N. 2013, Icarus, 225, 122

Kokubo, E., \& Genda, H. 2010, ApJ, 714, L21

Kokubo, E., \& Ida, S. 1998, Icarus, 131, 171

Kokubo, E., \& Ida, S. 2000, Icarus, 143, 15

Kokubo, E., Kominami, J., \& Ida, S. 2006, ApJ, 642, 1131

Laughlin, G., Steinacker, A., \& Adams, F. C. 2004, ApJ, 608, 489

Laskar, J. 1997, A\&A, 317, L75

Lega, E., Morbidelli, A., \& Nesvorný, D. 2013, MNRAS, 431, 3494

Levison, H. F., \& Agnor, C. 2003, AJ, 125, 2692

Levison, H. F., Morbidelli, A., Tsiganis, K., Nesvorný, D., \& Gomes, R. 2011, AJ, 142, 152 
Sofia Lykawka, P., \& Ito, T. 2013, arXiv:1306.3287

Lin, D. N. C., \& Papaloizou, J. 1986, ApJ, 309, 846

Lunine, J. I., Chambers, J., Morbidelli, A., \& Leshin, L. A. 2003, Icarus, 165, 1

Marty, B. \& Yokochi, R. 2006, Rev. mineral. Geochem., 62, 421

Marty, B. 2012, Earth and Planetary Science Letters, 313, 56

Morbidelli, A., Chambers, J., Lunine, J. I., et al. 2000, Meteoritics and Planetary Science, 35,1309

Morbidelli, A., \& Crida, A. 2007, Icarus, 191, 158

Morbidelli, A., Levison, H. F., Tsiganis, K., \& Gomes, R. 2005, Nature, 435, 462

Morbidelli, A., Lunine, J.I., O’Brien, D.P., Raymond, S.N., Walsh, K.J. 2012, Annual Reviews of Earth and Planetary Science, in press.

Morishima, R., Golabek, G. J., \& Samuel, H. 2013, Earth and Planetary Science Letters, 366,6

Morishima, R., Schmidt, M. W., Stadel, J., \& Moore, B. 2008, ApJ, 685, 1247

Nimmo, F., \& Kleine, T. 2007, Icarus, 191, 497

O’Brien, D. P., Morbidelli, A., \& Levison, H. F. 2006, Icarus, 184, 39

Papaloizou, J. C. B., \& Nelson, R. P. 2003, MNRAS, 339, 983

Petit, J.-M., Morbidelli, A., \& Chambers, J. 2001, Icarus, 153, 338

Pierens, A., \& Raymond, S. N. 2011, A\&A, 533, A131

Raymond, S. N., O’Brien, D. P., Morbidelli, A., \& Kaib, N. A. 2009, Icarus, 203, 644

Raymond, S. N., Quinn, T., \& Lunine, J. I. 2004, Icarus, 168, 1

Raymond, S. N., Quinn, T., \& Lunine, J. I. 2005, ApJ, 632, 670

Raymond, S. N., Quinn, T., \& Lunine, J. I. 2006, Icarus, 183, 265

Raymond, S. N., Quinn, T., \& Lunine, J. I. 2007, Astrobiology, 7, 66

Schlichting, H. E., Warren, P. H., \& Yin, Q.-Z. 2012, ApJ, 752, 8 
Thommes, E., Nagasawa, M., \& Lin, D. N. C. 2008, ApJ, 676, 728

Touboul, M., Kleine, T., Bourdon, B., Palme, H., \& Wieler, R. 2007, Nature, 450, 1206

Tsiganis, K., Gomes, R., Morbidelli, A., \& Levison, H. F. 2005, Nature, 435, 459

Usui, T., Alexander, C. M. O., Wang, J., Simon, J. I., \& Jones, J. H. 2012, Earth and Planetary Science Letters, 357, 119

Walsh, K. J., Morbidelli, A., Raymond, S. N., O’Brien, D. P., \& Mandell, A. M. 2011, Nature, 475, 206

Wetherill, G. W. 1996, Icarus, 119, 219

Yin, Q., Jacobsen, S. B., Yamashita, K., et al. 2002, Nature, 418, 949

This preprint was prepared with the AAS IATEX macros v5.2. 


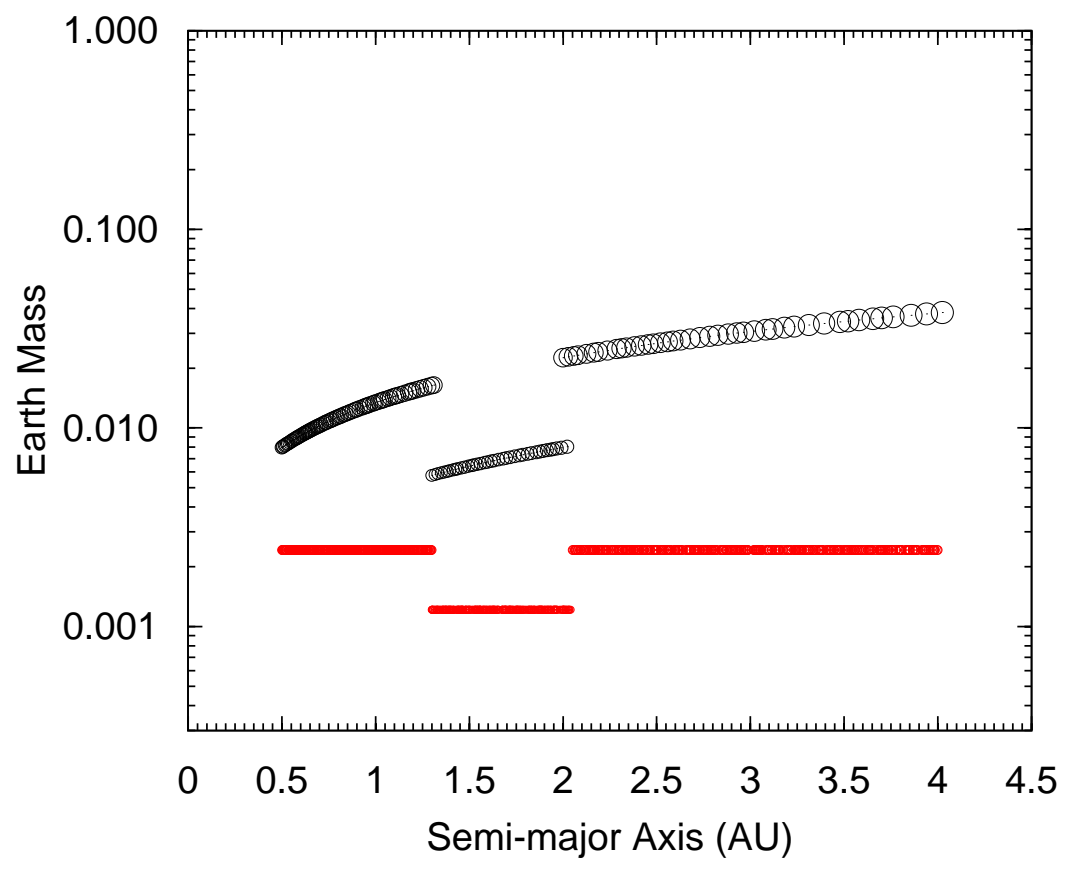

Fig. 1.- Initial distribution of 154 embryos (black) and 973 planetesimals (red) considering a mass-depletion of $50 \%$ extending from $1.3 \mathrm{AU}$ to $2.0 \mathrm{AU}$. The masses of planetesimals are smaller than 0.003 Earth masses. 


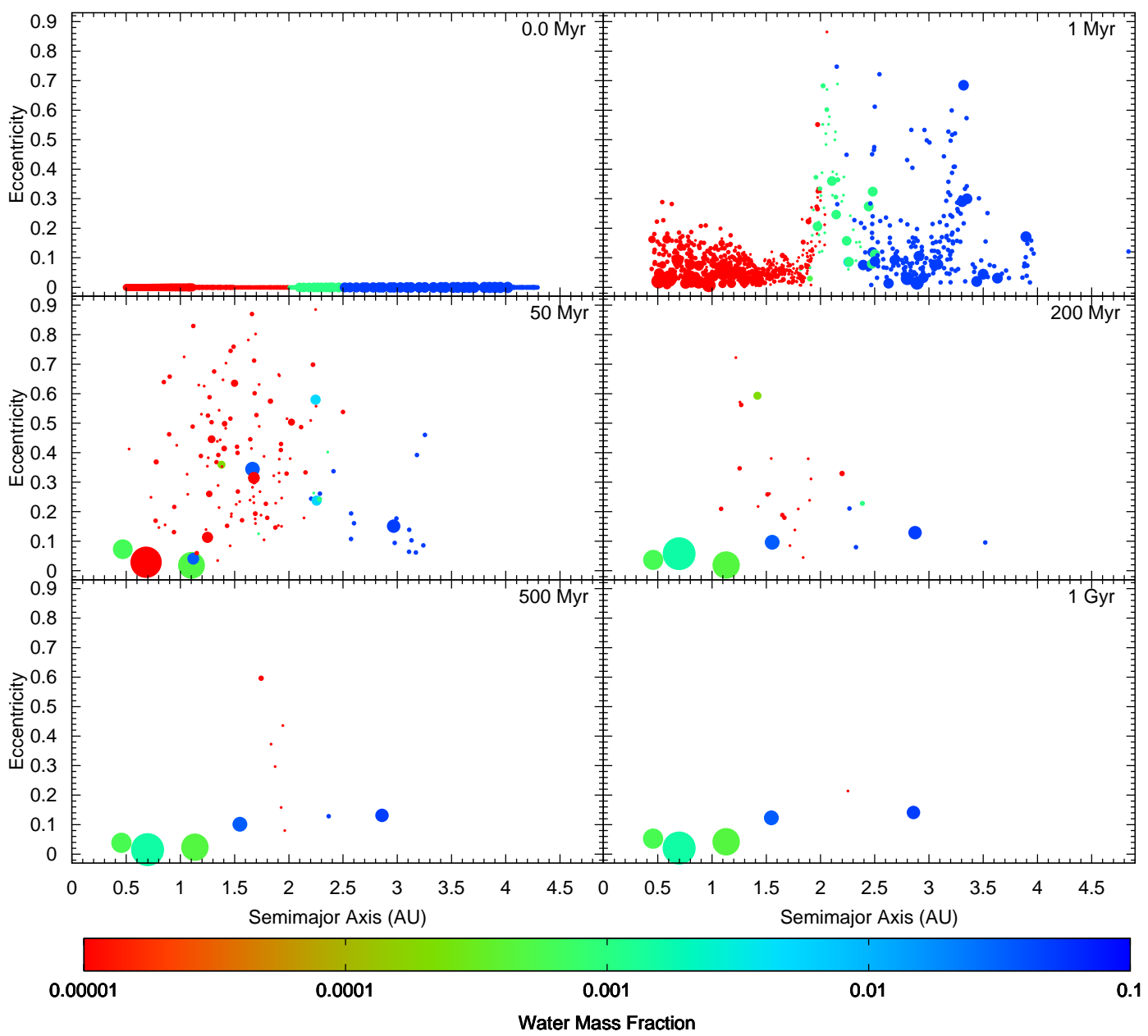

Fig. 2. - Snapshots of the formation and dynamical evolution of planets in a disk with a depletion of $75 \%$ extending from 1.1 AU to 2.1 AU. Jupiter and Saturn are in their current orbits. The size of each body corresponds to its relative physical size and is scaled as $M^{1 / 3}$. However, it is not to scale on the $x$-axis. The color-coding represents the water-mass fraction of the body. 


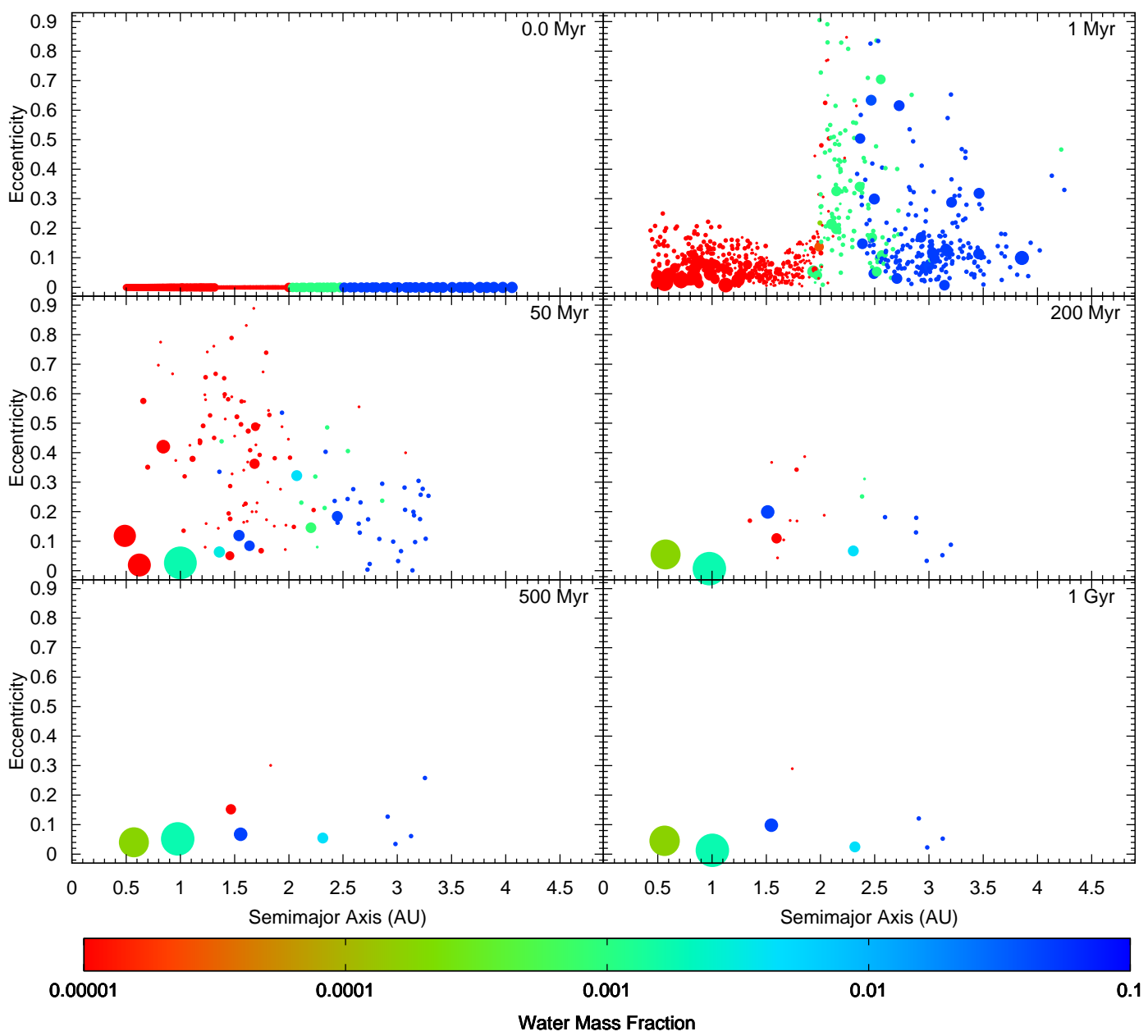

Fig. 3.- Snapshots of the formation and dynamical evolution of planets in a disk with a depletion of $75 \%$ extending from 1.3 AU to 2.0 AU. Jupiter and Saturn are in their current orbits. The size of each body corresponds to its relative physical size and is scaled as $M^{1 / 3}$. However, it is not to scale on the $x$-axis. The color-coding represents the water-mass fraction of the body. 


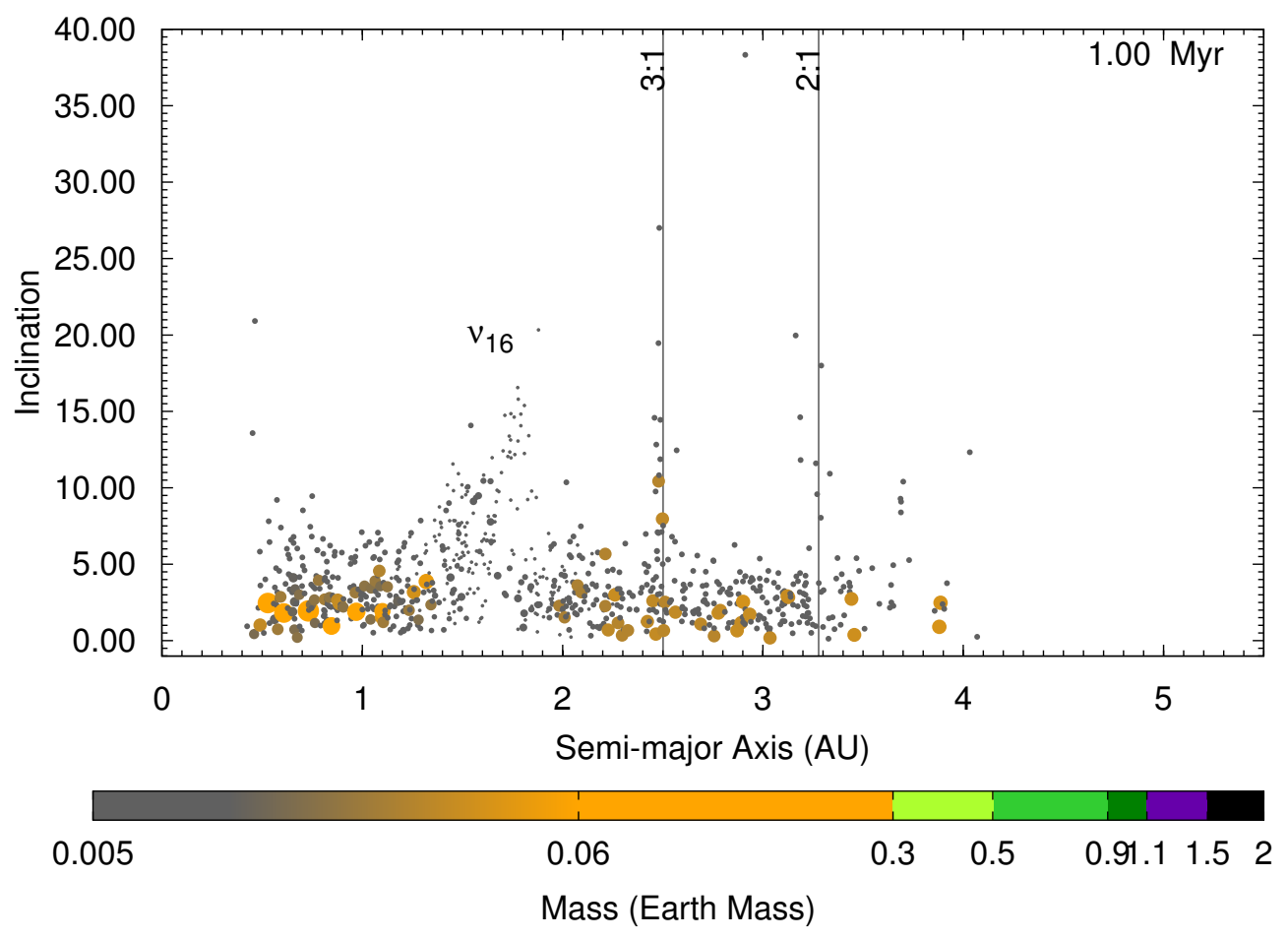

Fig. 4. - Graph of the orbital inclination versus semimajor axis for the first 1 Myr of the simulation of Figure 3. As shown here, $\nu_{16}$ and mean-motion resonances with Jupiter increase the inclinations of planetesimals and planetary embryos. This orbital excitation is more pronounced in the depleted region (1.3-2.1 AU). 


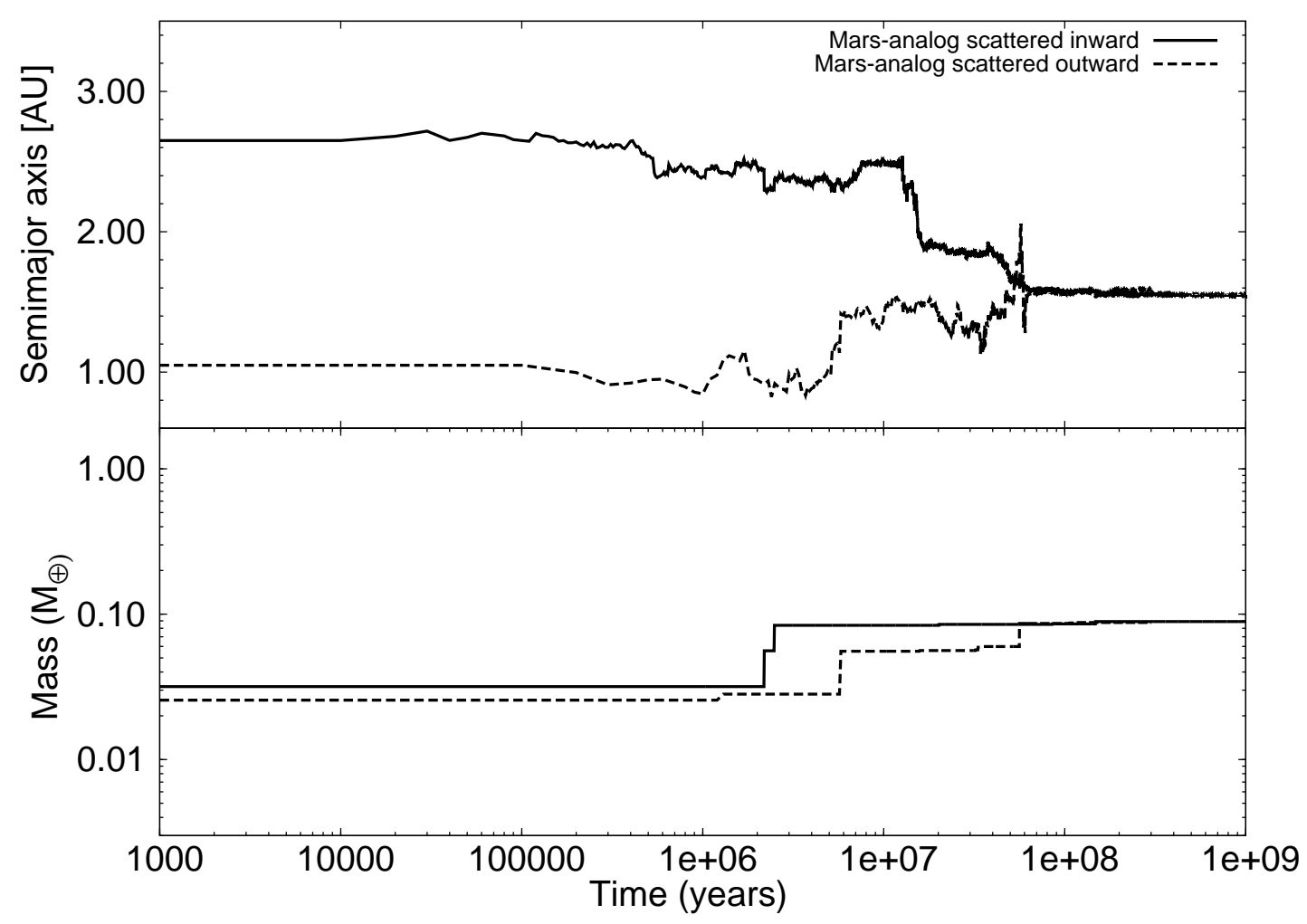

Fig. 5.- Graphs of the semimajor axes and masses of two typical Mars-analogs formed in simulations where Jupiter and Saturn were initially in their current orbits. Similar to the results shown here, Mars-analogs in all our simulations were formed in the inner or outer non-depleted regions and were scattered into the depleted area. 

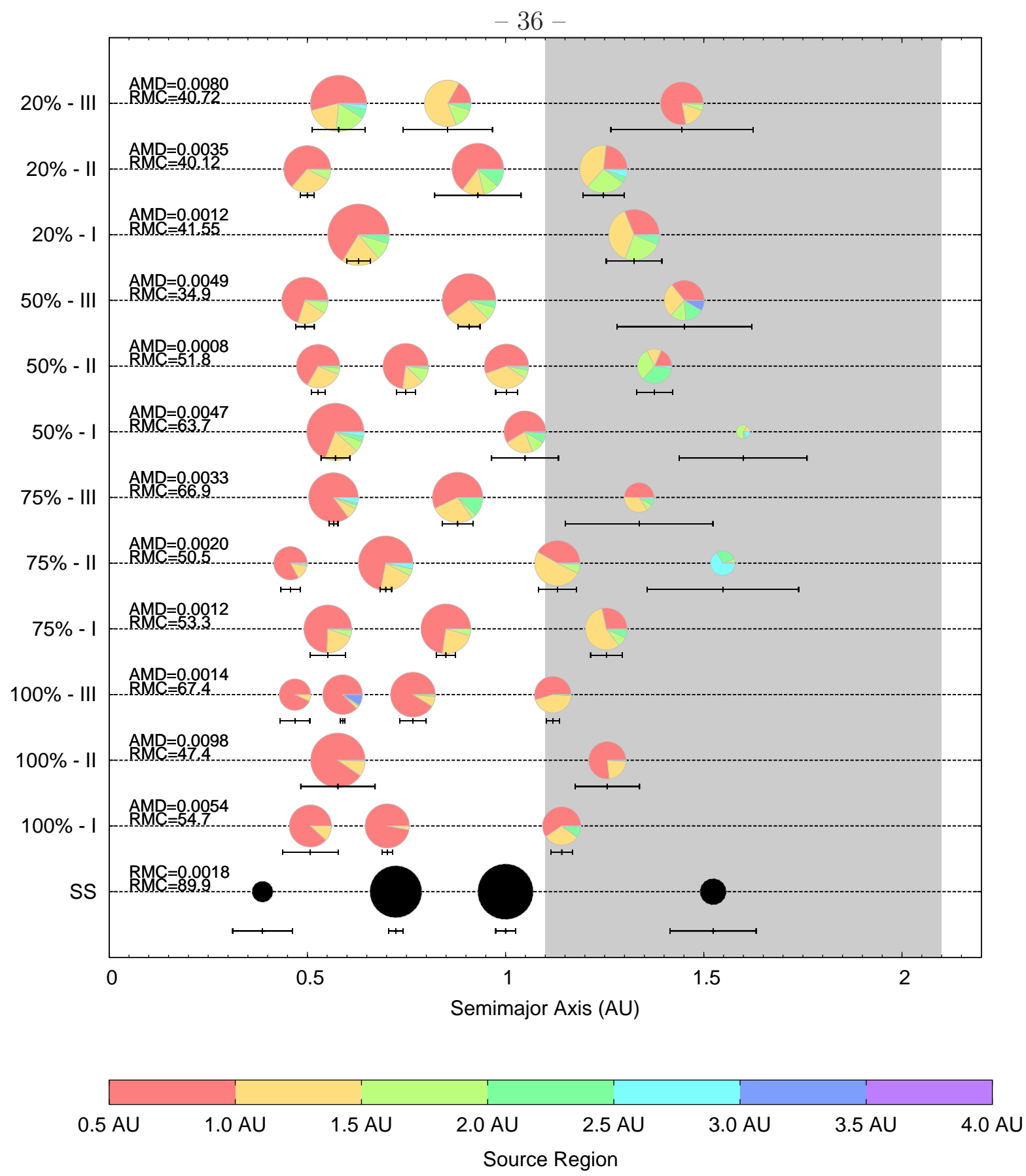

Fig. 6.- Final masses and orbital configurations of planets in simulations of disk model A with different mass-depletion scales. The depleted area is from 1.1 AU to 2.1 AU (shown in gray). As shown by the Roman numbers on the vertical axis, each simulation was run for three different distributions of planetesimals and planetary embryos. The size of each body corresponds to its relative physical size scaled as $M^{1 / 3}$. However, it is not to scale on the $x$-axis. The color of each object represents the relative contribution of material from different parts of the disk. The eccentricity of each planet is represented by its variation in heliocentric distance over the semimajor axis (horizontal bars). 

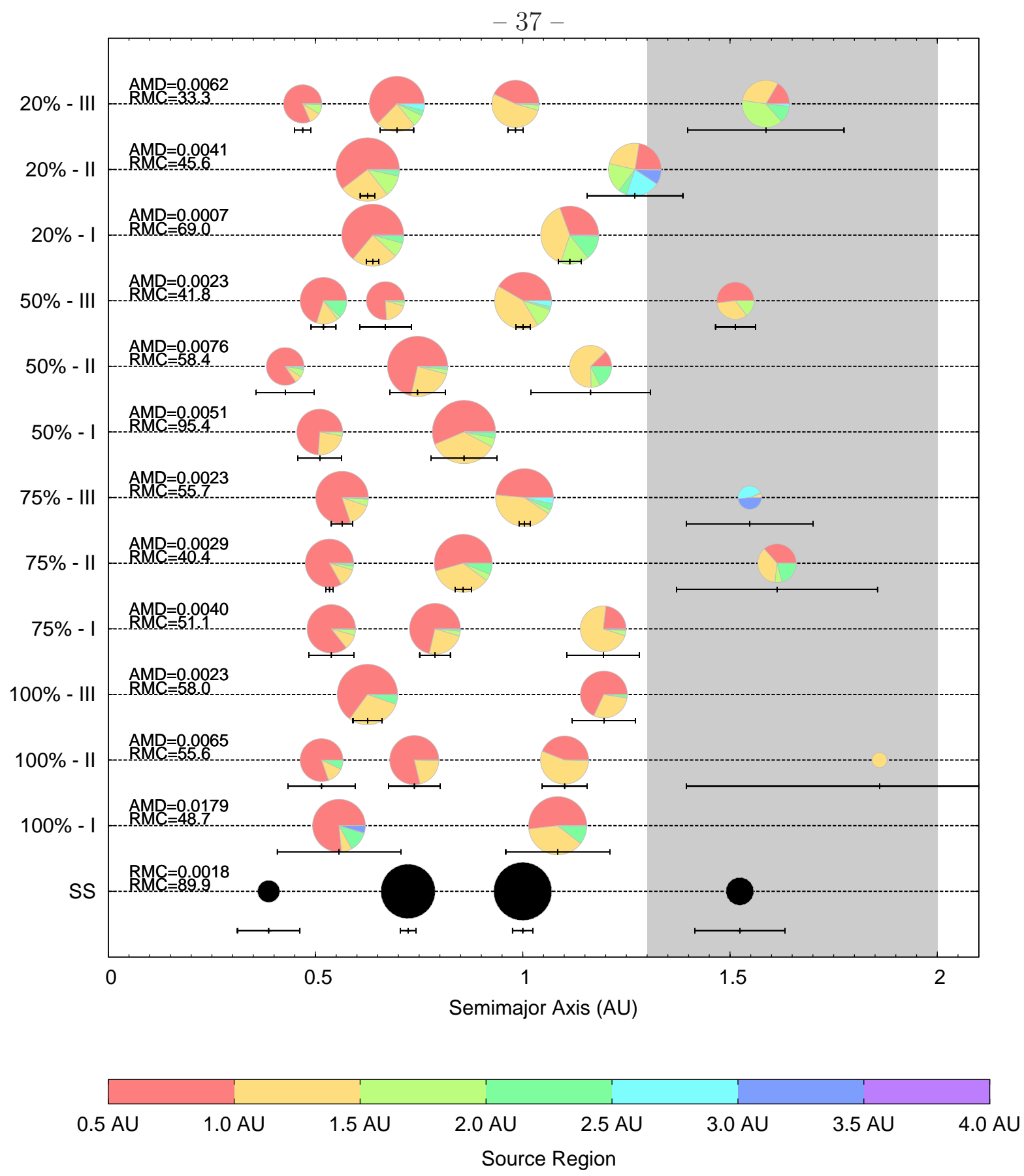

Fig. 7.- Final masses and orbital configurations of planets in simulations of disk model B with different mass-depletion scales. The depleted area is from 1.3 AU to 2.0 AU (shown in gray). As shown by the Roman numbers on the vertical axis, each simulation was run for three different distributions of planetesimals and planetary embryos. The size of each body corresponds to its relative physical size scaled as $M^{1 / 3}$. However, it is not to scale on the $x$-axis. The color of each object represents the relative contribution of material from different parts of the disk. The eccentricity of each planet is represented by its variation in heliocentric distance over the semimajor axis (horizontal bars). 

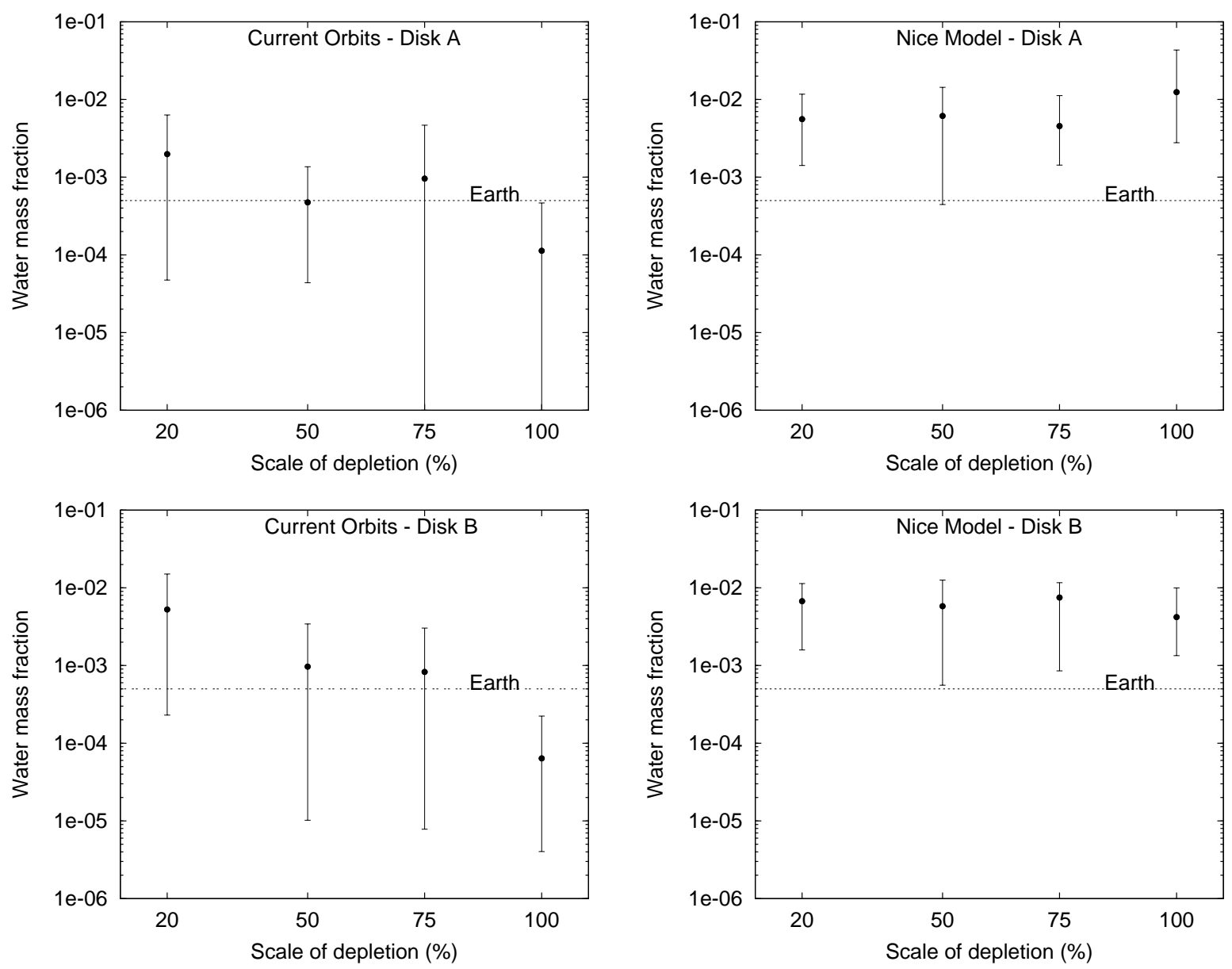

Fig. 8. - Graphs of the mean value of the water/mass fraction of the planets formed inside 1.3 AU as a function of the scale of the disk mass-depletion for different disk models and giant planets configuration. The panels on the left show the results of the simulations considering Jupiter and Saturn to be initially in their current orbits. The ones on the right show the results for which Jupiter and Saturn were initially as in the Nice Model (2005). 

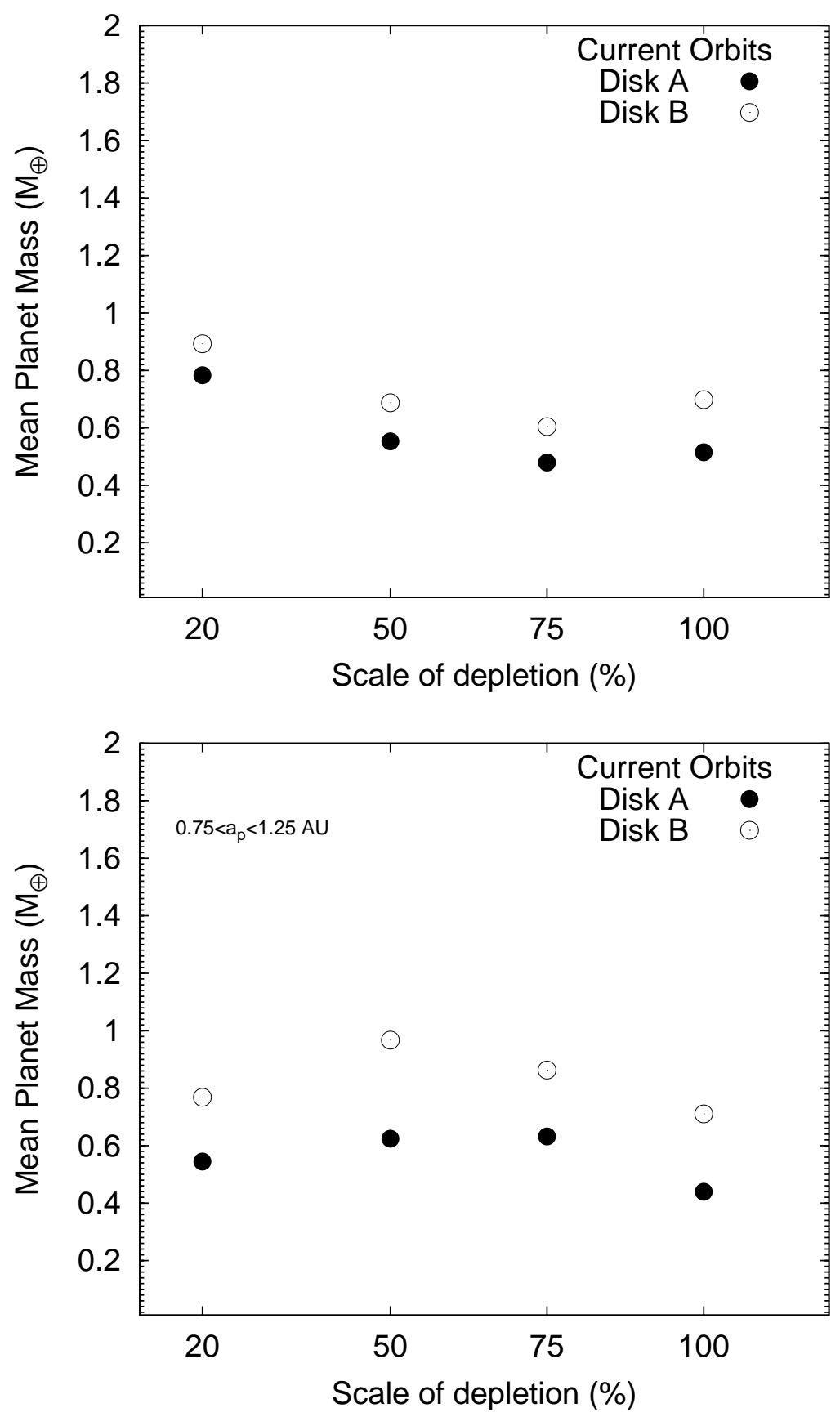

Fig. 9.- Graphs of the mean value of planet mass as a function of the scale of mass-depletion in different disk models. Jupiter and Saturn were initially in their current orbits. The top panel shows the mean planet mass for planets formed interior to $2 \mathrm{AU}$ and the bottom panel shows similar quantity for planets formed around $1 \mathrm{AU}\left(0.75<a_{p}<1.25 \mathrm{AU}\right)$. 


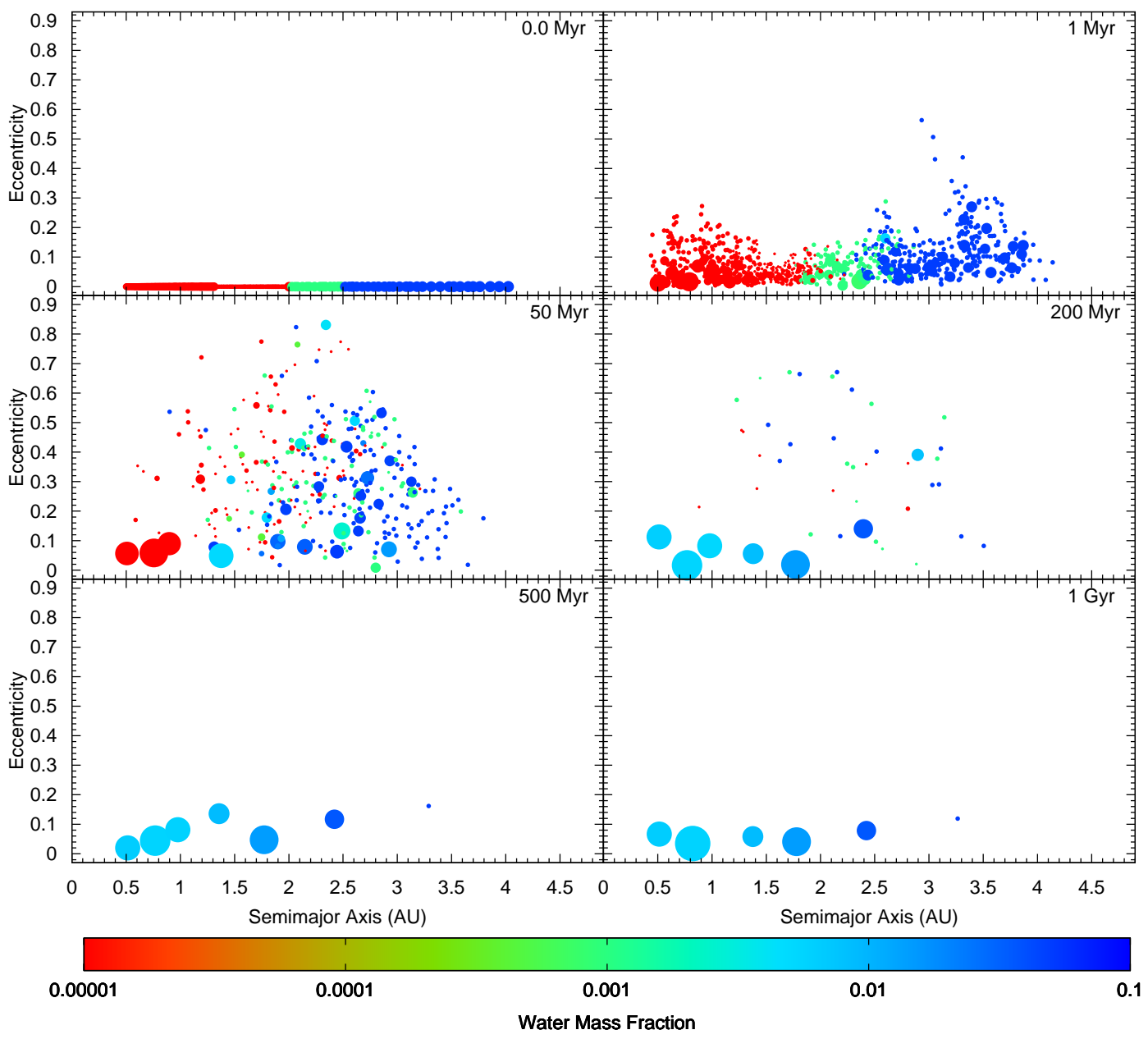

Fig. 10.- Snapshots of the formation and dynamical evolution of planets in a disk with a depletion of $75 \%$ extending from 1.3 AU to 2.0 AU. Jupiter and Saturn were initially in circular orbits corresponding to the Nice Model. The size of each body corresponds to its relative physical size and is scaled as $M^{1 / 3}$. However, it is not to scale on the $x$-axis. The color-coding represents the water-mass fraction of the body. 

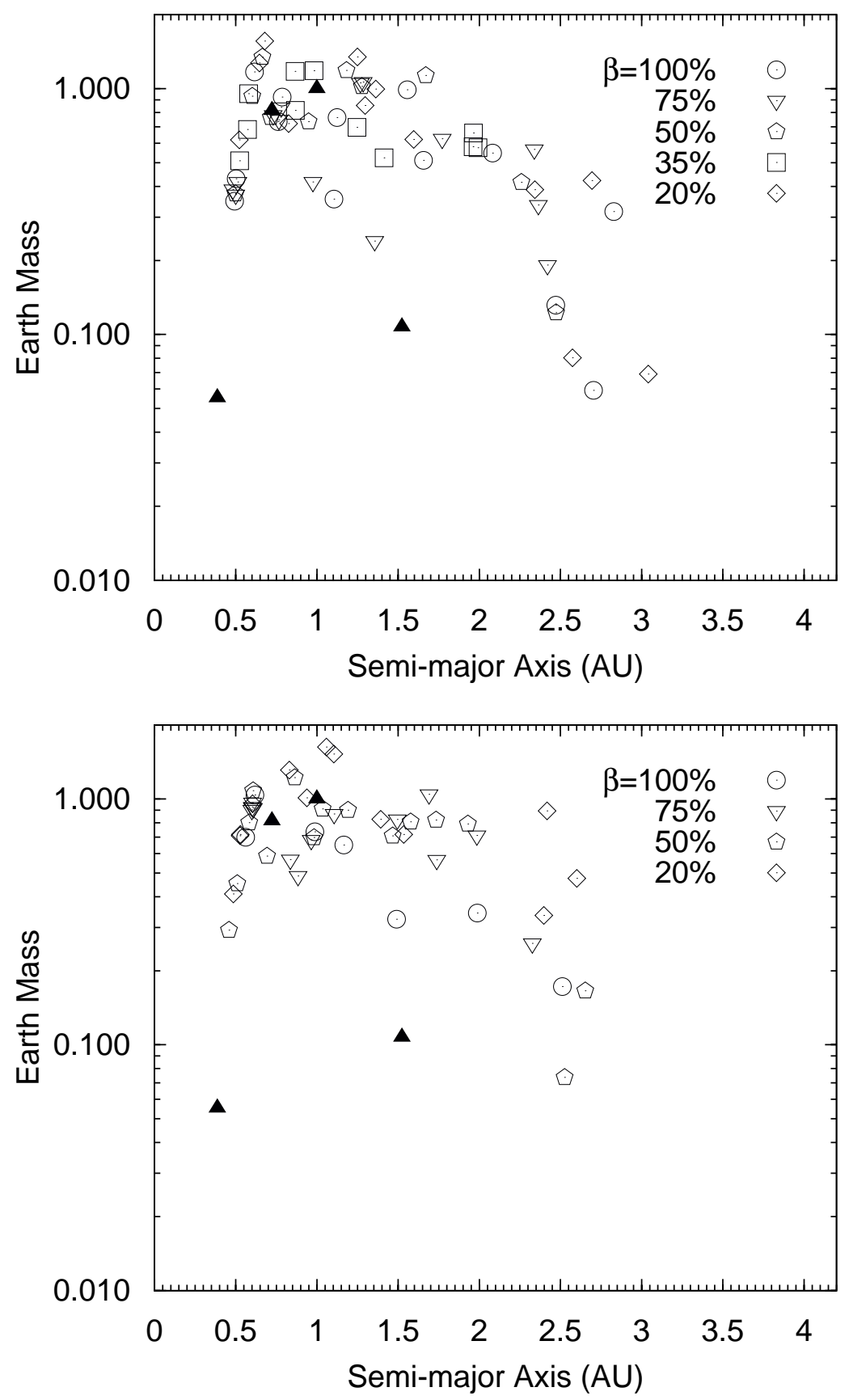

Fig. 11. - Graphs of the mass-semimajor axis distribution for the surviving bodies (open circles) in the simulations considering the disk model A (top) and disk model B (bottom) for all scales of depletion. Jupiter and Saturn were initially in circular orbits. The solid triangles represent the masses of Mars, Earth, Venus, and Mercury. The Surviving planetesimals are not shown. 

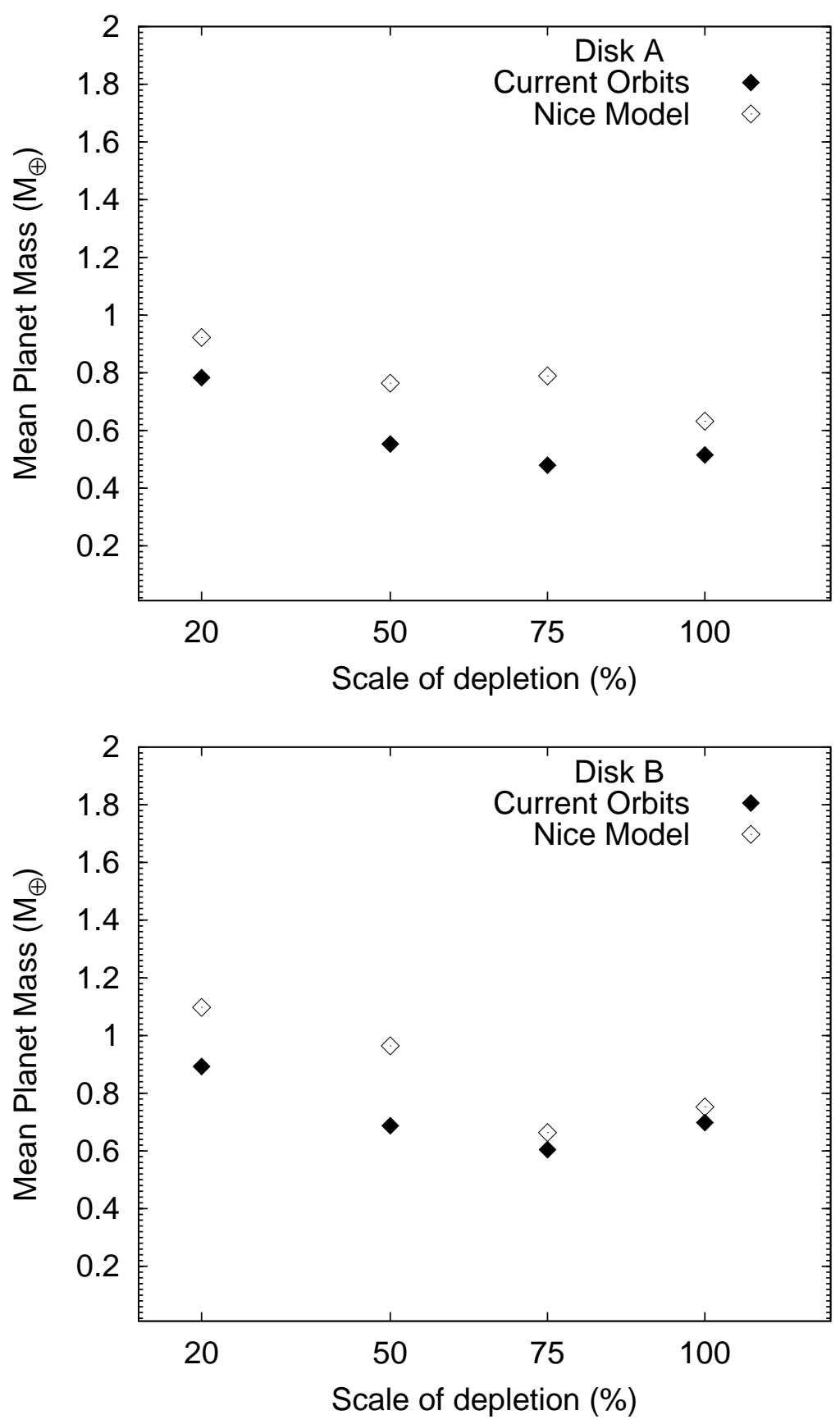

Fig. 12. - Graphs of the mean values of the masses of planets in terms of the scale of mass-depletion for different disk models and giant planets configurations. The top panel corresponds to the disk model A and the bottom panel is for the disk model B. 

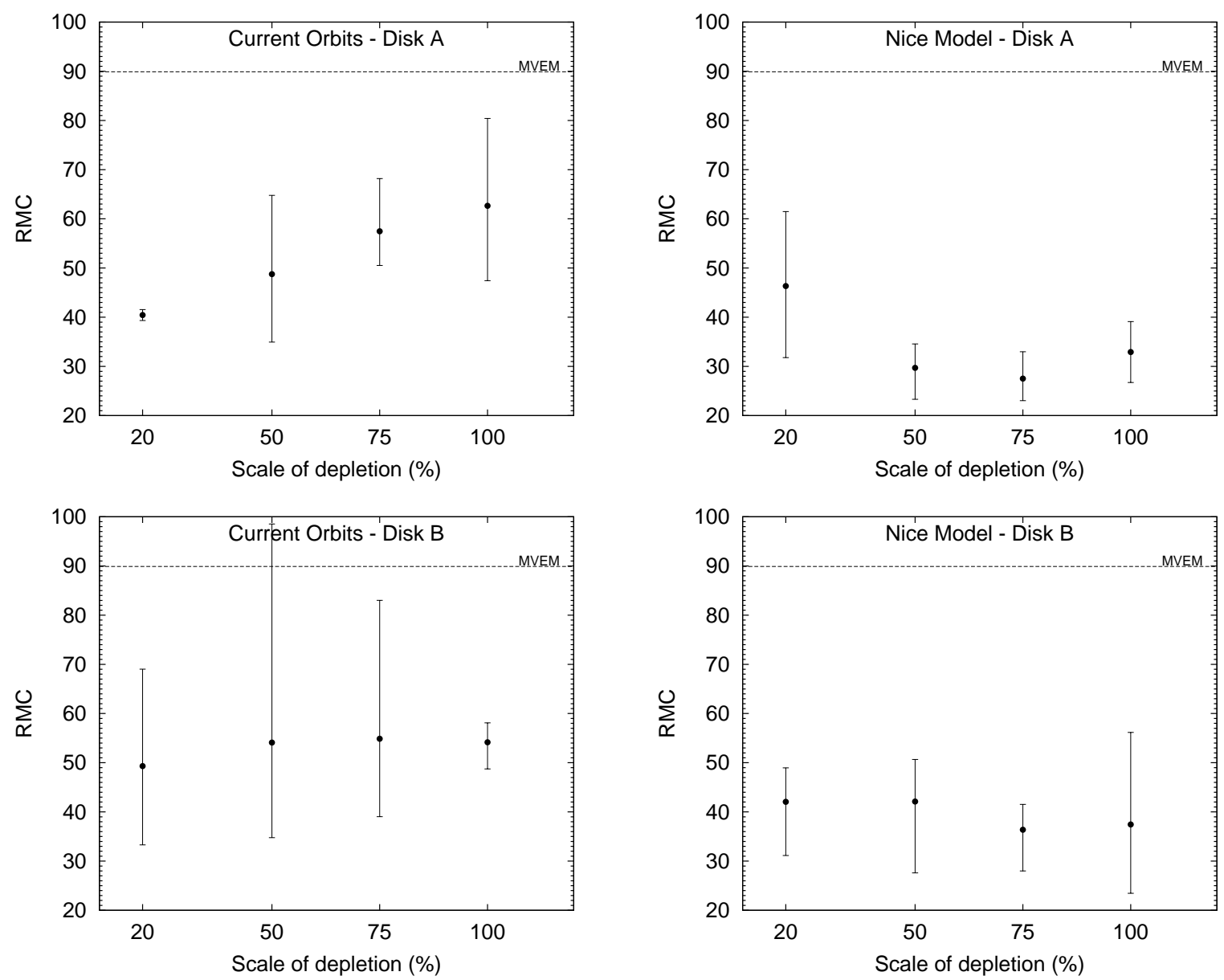

Fig. 13.- Graphs of the mean values of RMC as a function of the mass-depletion scale for different disk models and giant planets configuration. The left column shows the results for simulations in which Jupiter and Saturn were initially in their current orbits, and the right column shows the results when these planets were in circular orbits similar to those in the Nice model (2005). 

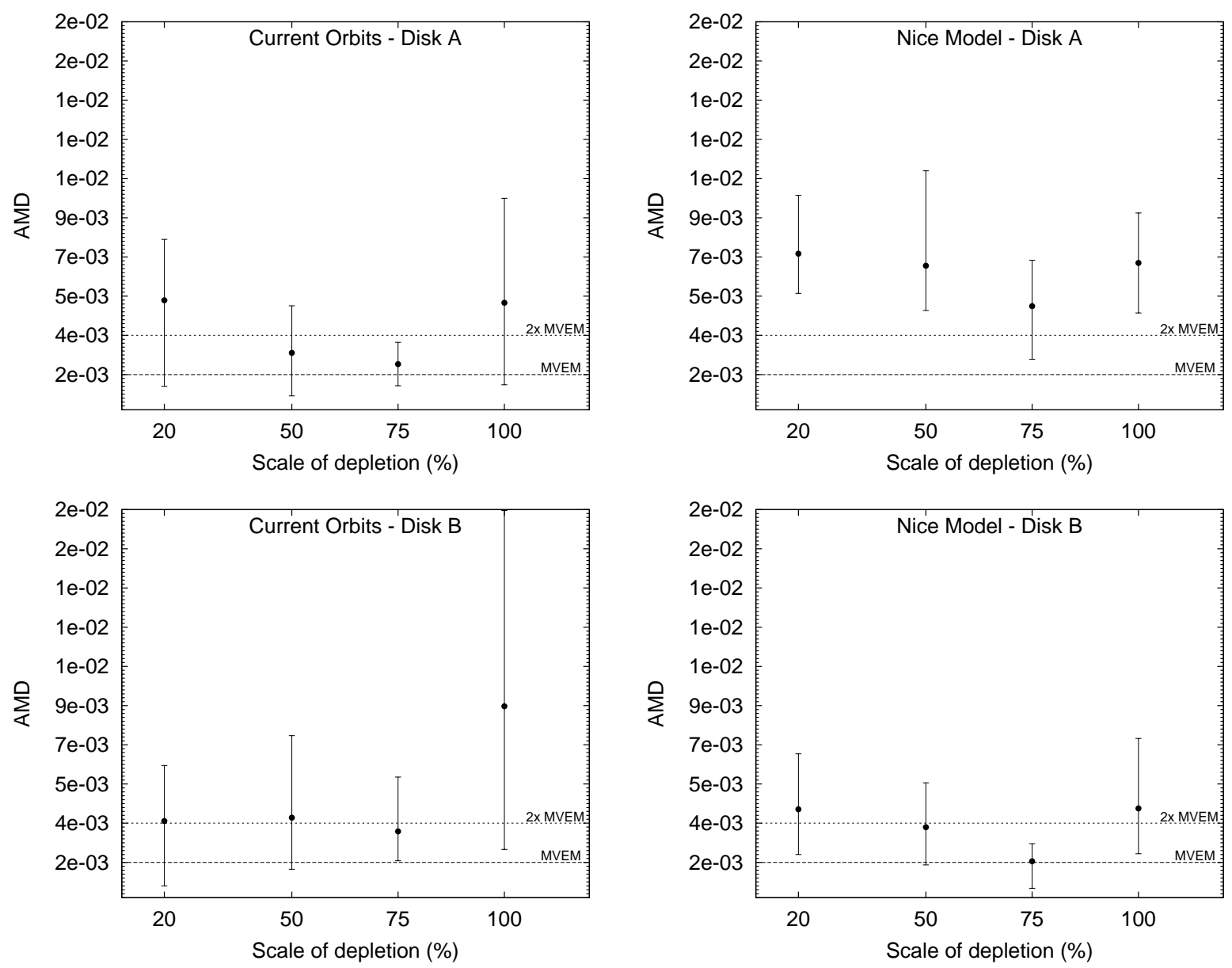

Fig. 14. - Graphs of the mean values of AMD as a function of the mass-depletion scale for different disk models and giant planets configuration. The left column shows the results for simulations in which Jupiter and Saturn were initially in their current orbits, and the right column shows the results when these planets were in circular orbits similar to those in the Nice model (2005). 

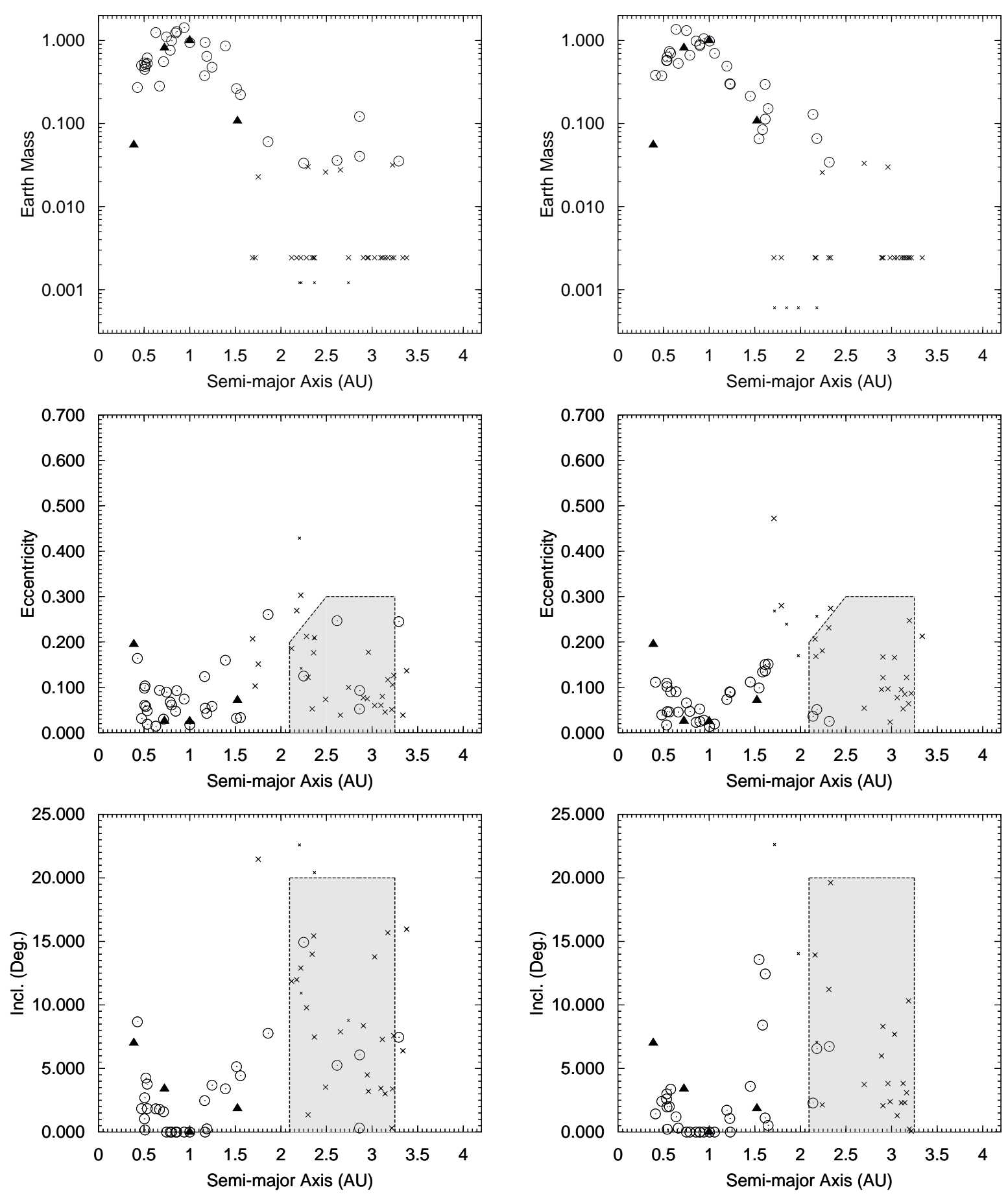

Fig. 15.- Orbital distributions of the surviving bodies in the simulations considering the disk model B with Jupiter and Saturn initially in their current orbits. Open circles correspond to bodies with masses larger than $0.3 M_{\text {Mars }} \approx 0.033 M_{\oplus}$. Smaller bodies are labeled with crosses. The left column shows the results of 9 simulations with a depletion scale of $50 \%$ and the right column corresponds to those with a depletion scale of $75 \%$. The solid triangles represent the inner planets of the solar system. The gray area shows the asteroid belt. 
Table 1: Regions and scales of mass-depletion

\begin{tabular}{lcc}
\hline Disk & Region $(\mathrm{AU})$ & Scale $(\%)$ \\
\hline \hline $\mathrm{A}$ & 1.1 to 2.1 & $20,50,75,100$ \\
\hline $\mathrm{B}$ & 1.3 to 2.0 & $20,35,50,75,100$ \\
\hline \hline
\end{tabular}


Table 2: Representative results of the simulations for the disk model A in which at least one body with a mass $M<0.5 M_{\oplus}$ was formed within $1.25 \mathrm{AU}$ and $2.0 \mathrm{AU}^{\mathrm{a}}$.

\begin{tabular}{|c|c|c|c|c|c|c|c|c|c|c|c|}
\hline$\overline{\overline{\text { Sim }}}$ & "Body & $\begin{array}{l}a_{\text {init }} \\
(\mathrm{AU}) \\
\end{array}$ & $\begin{array}{c}a_{\mathrm{fin}} \\
(\mathrm{AU}) \\
\end{array}$ & $\mathrm{e}$ & $\overline{\mathrm{II}\left({ }^{\circ}\right)}$ & $\begin{array}{l}\text { Mass } \\
\left(M_{\oplus}\right) \\
\end{array}$ & $\overline{\overline{W M F}}$ & $\overline{N_{c}}$ & 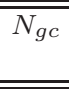 & $\begin{array}{l}t_{50 \%} \\
(\mathrm{Myr}) \\
\end{array}$ & $\begin{array}{l}t_{90 \%} \\
(\mathrm{Myr}) \\
\end{array}$ \\
\hline$\overline{\mathrm{A}-100 \%-\mathrm{II}}$ & $\overline{M 17}$ & 0.5988 & 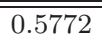 & 0.1626 & 11.9910 & 0.9479 & $2.581546 \mathrm{e}-06$ & "43 & 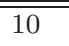 & 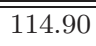 & 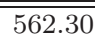 \\
\hline A-100\%- II & M36 & .7690 & 1.2567 & 0.0647 & 9.9490 & 0.3066 & $7.981451 \mathrm{e}-06$ & 25 & 8 & 3.61 & 116.70 \\
\hline A- $100 \%-$ II & EM93 & 3.5587 & 2.6544 & 0.2764 & 4.0460 & 0.0667 & $5.000000 \mathrm{e}-02$ & 1 & 1 & 3.40 & 3.40 \\
\hline A-75\%- I & EM5 & 5207 & 0.5516 & 0.0802 & 3.6940 & .6412 & $2.013308 \mathrm{e}-04$ & 87 & 10 & 32.83 & 138.90 \\
\hline A-75\%- I & M52 & 8923 & 0.8495 & 0.0288 & 2.9710 & .7274 & $0.00000 \mathrm{e}+00$ & 91 & 12 & 39.44 & 53.38 \\
\hline A-75\%- I & EM64 & 1.0636 & 1.2543 & 0.0318 & 2.7140 & 0.4355 & $3.537550 \mathrm{e}-04$ & 33 & 6 & 62.42 & 147.70 \\
\hline A-75\%- I & M164 & 2.3522 & 2.2403 & 0.1213 & 3.2200 & .0256 & $1.000000 \mathrm{e}-03$ & 0 & 0 & 0.00 & 0.00 \\
\hline A-75\%- I & EM173 & 2.7681 & 3.0647 & 0.0698 & 2.9860 & 0.0660 & $4.907275 \mathrm{e}-02$ & 6 & 1 & 0.00 & 0.00 \\
\hline$\overline{\mathrm{A}-75 \%-\mathrm{II}}$ & EM34 & 0.7457 & 0.4576 & 0.0528 & 4.2490 & 0.2195 & $5.723449 \mathrm{e}-04$ & 40 & 6 & 4.05 & 40.76 \\
\hline A-75\%- II & M16 & 0.5941 & 0.6979 & 0.0212 & 3.5730 & 0.9520 & $1.572659 \mathrm{e}-03$ & 99 & 15 & 12.34 & 64.75 \\
\hline A-75\%- II & M53 & 0.9574 & 1.1308 & 0.0423 & 3.5950 & .5470 & 4.593763 & 63 & 13 & 12.92 & 28.36 \\
\hline A-75\%- II & EM167 & 2.6842 & 1.5480 & 0.1236 & 13.4810 & 0.0864 & $3.373369 \mathrm{e}-02$ & 7 & - & 2.19 & 2.48 \\
\hline A-75\%- II & EM172 & 2.9346 & 2.8572 & 0.1416 & 6.4470 & 0.0645 & $4.952557 \mathrm{e}-02$ & 4 & 1 & 0.60 & 0.60 \\
\hline A-75\%- III & EM12 & 0.5698 & 0.5661 & 0.0197 & 1.1750 & 0.7136 & $1.903639 \mathrm{e}-03$ & 54 & 11 & 86.33 & 537.20 \\
\hline A-75\%- III & M34 & 0.7334 & 0.8792 & 0.0439 & 6.1250 & 0.7475 & 1.22719 & 77 & 14 & 12.94 & 21.26 \\
\hline A-75\%- III & EM45 & 0.8446 & 1.3370 & 0.1392 & 8.7110 & 0.1563 & $8.532762 \mathrm{e}-04$ & 23 & 4 & 56.91 & 74.10 \\
\hline A-75\%- III & EM161 & 2.2421 & 2.2601 & 0.0261 & 0.5690 & 0.0578 & $9.788227 \mathrm{e}-04$ & 6 & 1 & 0.00 & 0.00 \\
\hline$\overline{A-50 \%-I}$ & EM19 & 0.6052 & 0.5708 & 0.0638 & 2.8780 & 1.0949 & $1.310486 \mathrm{e}-03$ & 82 & 20 & 52.35 & 163.60 \\
\hline A-50\%- I & EM42 & 0.7977 & 1.0492 & 0.0803 & 3.7560 & 0.4318 & $6.430292 \mathrm{e}-04$ & 41 & 11 & 10.94 & 71.66 \\
\hline A-50\%- I & EM113 & 1.7161 & 1.5994 & 0.1008 & 29.1090 & 0.0157 & $7.859689 \mathrm{e}-03$ & 6 & 0 & 0.00 & 40.56 \\
\hline A-50\%- I & EM169 & 3.9663 & 3.9013 & 0.1441 & 1.9040 & 0.0379 & $5.000000 \mathrm{e}-02$ & 0 & 0 & 0.00 & 0.00 \\
\hline$\overline{\mathrm{A}-50 \%-\mathrm{II}}$ & EM4 & 0.5167 & 0.5274 & 0.0329 & 0.9110 & 0.4821 & $1.522684 \mathrm{e}-05$ & 63 & 9 & 20.48 & 53.26 \\
\hline A-50\%- II & EM45 & 0.8718 & 0.7484 & 0.0320 & 3.6580 & 0.5544 & $2.354406 \mathrm{e}-04$ & 80 & 13 & 3.17 & 54.09 \\
\hline A-50\%- II & EM47 & 0.8949 & 1.0026 & 0.0275 & 3.9390 & 0.5127 & $2.640177 \mathrm{e}-04$ & 57 & 13 & 5.52 & 43.28 \\
\hline A-50\%- II & EM128 & 2.1000 & 1.3759 & 0.0332 & 5.4550 & 0.2402 & $1.363240 \mathrm{e}-03$ & 19 & 7 & 28.94 & 216.20 \\
\hline A-50\%- II & EM148 & 3.0285 & 3.0188 & 0.1541 & 6.0760 & 0.0359 & $5.000000 \mathrm{e}-02$ & 2 & 0 & 0.00 & 0.00 \\
\hline$\overline{\mathrm{A}-50 \%-\mathrm{IV}}$ & EM18 & 0.6036 & 0.4939 & 0.0481 & 8.6880 & 0.5856 & $2.151852 \mathrm{e}-04$ & $\overline{78}$ & $\overline{14}$ & 4.18 & $\overline{44.41}$ \\
\hline A-50\%- IV & EM16 & 0.5941 & 0.9080 & 0.0303 & 1.1200 & 0.9066 & $4.376598 \mathrm{e}-05$ & 70 & 17 & 58.97 & 86.67 \\
\hline A-50\%- IV & EM133 & 2.3435 & 1.4509 & 0.1171 & 4.8840 & 0.3826 & $4.400611 \mathrm{e}-03$ & 15 & 5 & 53.69 & 126.40 \\
\hline
\end{tabular}

${ }^{a}$ From left to right, the columns show the simulation, the body, the semimajor axis of body in the beginning of the simulation, its final semimajor axis, eccentricity, inclination (deg), water-mass fraction, total number of collisions, number of collisions with large objects (impactor mass $>0.01 M_{\oplus}$ ), time of reaching $50 \%$ of the mass, and time of reaching $90 \%$ of the mass, respectively. 
Table 3: Representative results of the simulations for the disk model B in which at least one body with a mass $M<0.5 M_{\oplus}$ was formed within $1.25 \mathrm{AU}$ and $2.0 \mathrm{AU}^{\mathrm{a}}$.

\begin{tabular}{|c|c|c|c|c|c|c|c|c|c|c|c|}
\hline Sim & Body & $\begin{array}{l}a_{\text {init }} \\
(\mathrm{AU})\end{array}$ & $\begin{array}{c}a_{f i n} \\
(\mathrm{AU}) \\
\end{array}$ & $\mathrm{e}$ & $\mathrm{I}\left({ }^{\circ}\right)$ & $\begin{array}{l}\text { Mass } \\
\left(M_{\oplus}\right) \\
\end{array}$ & WMF & $N_{c}$ & $N_{g c}$ & $\begin{array}{l}t_{50 \%} \\
\text { (Myr) }\end{array}$ & $\begin{array}{l}t_{90 \%} \\
(\mathrm{Myr}) \\
\end{array}$ \\
\hline B-75\%- II & EM26 & 0.6646 & 0.5338 & 0.0169 & 2.6090 & 0.5749 & $1.808761 \mathrm{e}-05$ & 88 & 14 & 2.60 & 41.26 \\
\hline B-75\%- II & EM60 & 287 & 3565 & 0229 & 5.2200 & 9882 & 6.24239 & 61 & 14 & 6 & 49.60 \\
\hline B-75\%- II & EM70 & 1.1774 & 1.6135 & 0.1501 & 4.1080 & 0.2976 & $2.068806 \mathrm{e}-04$ & 27 & 9 & 1.85 & 103.30 \\
\hline B-75\%- III & EM16 & 0.5973 & 0.5641 & 0.0457 & 5.3410 & 0.7419 & $1.715158 \mathrm{e}-04$ & 58 & 8 & 54.60 & 93.92 \\
\hline B-75\%- III & EM5 & 0.5233 & 1.0044 & 0.0135 & 3.3400 & 0.9884 & $1.789666 \mathrm{e}-03$ & 81 & 18 & 6.23 & 31.69 \\
\hline B-75\%- III & EM159 & 3.1186 & 1.5477 & 0.0987 & 16.9160 & 0.0658 & 4.674 & 3 & 1 & & 5.59 \\
\hline B-75\%- III & EM137 & 2.1413 & 2.3173 & 0.0251 & 10.0770 & 0.0345 & $4.380566 \mathrm{e}-03$ & 5 & 0 & 0.00 & 7.03 \\
\hline B-50\%- II & EM12 & 0.5742 & 0.4269 & 0.1639 & 10.8200 & 0.2736 & $4.650020 \mathrm{e}-04$ & 37 & 6 & 24.02 & 73.82 \\
\hline B-50\%- II & EM19 & 0.6214 & 0.7462 & 0.0894 & 2.1440 & 1.1127 & 2.3157 & 93 & 17 & 19.00 & 4.39 \\
\hline B-50\%- II & EM64 & 1.0799 & 1.1638 & 0.1237 & 4.6200 & 0.3797 & 1.764 & 22 & 7 & & 70.54 \\
\hline B-50\%- II & EM136 & 2.6272 & 2.6524 & 0.0389 & 10.0330 & 0.0279 & $5.000000 \mathrm{e}-02$ & 0 & 0 & 0.00 & 0.00 \\
\hline B-50\%- III & EM22 & 0.6366 & 0.5194 & 0.0577 & 6.0580 & 0.5161 & $7.913091 \mathrm{e}-05$ & 69 & 10 & 5.34 & 61.23 \\
\hline B-50\%- III & EM25 & 0.6628 & 0.6688 & 0.0933 & 3.5970 & 0.2841 & $1.291794 \mathrm{e}-05$ & 46 & 7 & 3.90 & 45.56 \\
\hline B-50\%- III & EM34 & 96 & 1.0009 & 0.0174 & 1.8090 & 0.9474 & 1.656 & 85 & 15 & & 116.50 \\
\hline B-50\%- III & EM10 & 0.5609 & 1.5132 & 0.0319 & 6.9570 & 0.2653 & $0.000000 \mathrm{e}+00$ & 27 & 7 & 28.67 & 229.90 \\
\hline B-50\%- III & EM127 & 2.4728 & 2.8621 & 0.0521 & 1.4900 & 0.1222 & $2.729102 \mathrm{e}-02$ & 5 & 3 & 1.58 & 25.55 \\
\hline B-35\%- I & EM38 & 0.7734 & 0.5684 & 0.0218 & 1.2620 & 0.8535 & $3.620376 \mathrm{e}-04$ & 88 & 18 & & 50.99 \\
\hline B-35\%- I & EM11 & 0.5580 & 0.9886 & 0.0331 & 6.3010 & 0.9829 & $2.852670 \mathrm{e}-04$ & 66 & 18 & & 92.21 \\
\hline B-35\%- I & EM53 & 0.9462 & 1.6636 & 0.1205 & 5.6370 & 0.3405 & $5.966567 \mathrm{e}-04$ & 24 & 6 & 25.49 & 52.22 \\
\hline
\end{tabular}

${ }^{a}$ From left to right, the columns show the simulation, the body, the semimajor axis of body in the beginning of the simulation, its final semimajor axis, eccentricity, inclination (deg), water-mass fraction, total number of collisions, number of collisions with large objects (impactor mass $>0.01 M_{\oplus}$ ), time of reaching $50 \%$ of the mass, and time of reaching $90 \%$ of the mass, respectively. 
Table 4: Summary of the results of the simulations in disk model A. The heading of each column represents the criterion that was used for assessing the success of a simulation. A success is indicated by $(\checkmark)$, a failure is shown by $(\times)$, and a $(\sim)$ indicates a near successful case $^{\text {a }}$

\begin{tabular}{lcccccccc}
\hline Sim. & $M_{\text {Mars }}$ & $t_{\text {form,Mars }}$ & $M_{\text {Earth }}$ & $t_{\text {form,Earth }}$ & $W M F_{\text {Earth }}$ & AMD & $M_{\text {ast }}$ & $N_{\text {ast }}$ \\
\hline A-75\%- I & $\times$ & $\times$ & $\checkmark$ & $\checkmark$ & $\times$ & $\checkmark$ & $\times$ & 0 \\
\hline A-75\% - II & $\checkmark$ & $\checkmark$ & $\sim$ & $\sim$ & $\sim$ & $\checkmark$ & $\times$ & 1 \\
\hline A-75\% - III & $\checkmark$ & $\times$ & $\checkmark$ & $\times$ & $\times$ & $\checkmark$ & $\checkmark$ & 6 \\
\hline A-50\%- I & $\times$ & $\times$ & $\times$ & $\times$ & $\times$ & $\times$ & $\checkmark$ & 1 \\
\hline A-50\% - II & $\checkmark$ & $\times$ & $\times$ & $\times$ & $\times$ & $\checkmark$ & $\checkmark$ & 4 \\
\hline A-50\%- III & $\times$ & $\times$ & $\checkmark$ & $\checkmark$ & $\times$ & $\times$ & $\checkmark$ & 9 \\
\hline \hline
\end{tabular}

${ }^{a}$ From left to right the columns represent the simulation, mass of the Mars-analog candidate, timescale of the formation of the Mars-analog candidate, mass of the Earth candidate, timescale of the formation of the Earth candidate, water-mass fraction of the Earth candidate, angular momentum deficit of the system (AMD), mass in embryos stranded in the asteroid belt, and the number of asteroids left. 
Table 5: Summary of the results of the simulations in disk model B. The heading of each column represents the criterion that was used for assessing the success of a simulation. A success is indicated by $(\checkmark)$, a failure is shown by $(\times)$, and a $(\sim)$ indicates a near successful $\operatorname{case}^{\mathrm{a}}$

\begin{tabular}{lcccccccc}
\hline Sim. & $M_{\text {Mars }}$ & $t_{\text {form,Mars }}$ & $M_{\text {Earth }}$ & $t_{\text {form,Earth }}$ & $W M F_{\text {Earth }}$ & AMD & $M_{\text {ast }}$ & $N_{\text {ast }}$ \\
\hline \hline B-75\% - I & $\times$ & $\times$ & $\checkmark$ & $\times$ & $\times$ & $\times$ & $\checkmark$ & 2 \\
\hline B-75\% - II & $\checkmark$ & $\times$ & $\checkmark$ & $\checkmark$ & $\times$ & $\checkmark$ & $\checkmark$ & 0 \\
\hline B-75\% - III & $\checkmark$ & $\times$ & $\checkmark$ & $\checkmark$ & $\checkmark$ & $\checkmark$ & $\checkmark$ & 3 \\
\hline B-75\% - IV & $\checkmark$ & $\times$ & $\checkmark$ & $\checkmark$ & $\checkmark$ & $\checkmark$ & $\checkmark$ & 1 \\
\hline B-75\% - V & $\checkmark$ & $\times$ & $\checkmark$ & $\checkmark$ & $\checkmark$ & $\times$ & $\times$ & 1 \\
\hline B-75\% - VI & $\checkmark$ & $\times$ & $\checkmark$ & $\times$ & $\times$ & $\checkmark$ & $\checkmark$ & 2 \\
\hline B-75\% - VII & $\times$ & $\times$ & $\checkmark$ & $\checkmark$ & $\checkmark$ & $\times$ & $\checkmark$ & 10 \\
\hline B-75\% - VIII & $\checkmark$ & $\times$ & $\checkmark$ & $\times$ & $\times$ & $\checkmark$ & $\times$ & 0 \\
\hline B-75\% - IX & $\times$ & $\times$ & $\times$ & $\times$ & $\times$ & $\times$ & $\checkmark$ & 0 \\
\hline B-50\%- I & $\times$ & $\times$ & $\checkmark$ & $\times$ & $\times$ & $\times$ & $\checkmark$ & 4 \\
\hline B-50\%- II & $\times$ & $\times$ & $\times$ & $\times$ & $\times$ & $\times$ & $\checkmark$ & 1 \\
\hline B-50\%- III & $\checkmark$ & $\times$ & $\checkmark$ & $\checkmark$ & $\checkmark$ & $\checkmark$ & $\times$ & 2 \\
\hline B-50\% - IV & $\checkmark$ & $\times$ & $\checkmark$ & $\checkmark$ & $\checkmark$ & $\checkmark$ & $\checkmark$ & 3 \\
\hline B-50\% - V & $\times$ & $\times$ & $\times$ & $\times$ & $\times$ & $\times$ & $\checkmark$ & 2 \\
\hline B-50\% - VI & $\checkmark$ & $\checkmark$ & $\checkmark$ & $\sim$ & $\checkmark$ & $\times$ & $\checkmark$ & 2 \\
\hline B-50\% - VII & $\times$ & $\times$ & $\checkmark$ & $\times$ & $\times$ & $\checkmark$ & $\times$ & 8 \\
\hline B-50\% - VIII & $\checkmark$ & $\times$ & $\checkmark$ & $\checkmark$ & $\times$ & $\checkmark$ & $\checkmark$ & 0 \\
\hline B-50\% - IX & $\times$ & $\times$ & $\checkmark$ & $\checkmark$ & $\checkmark$ & $\checkmark$ & $\checkmark$ & 2 \\
\hline
\end{tabular}

${ }^{b}$ From left to right the columns represent the simulation, mass of the Mars-analog candidate, timescale of the formation of the Mars-analog candidate, mass of the Earth candidate, timescale of the formation of the Earth candidate, water-mass fraction of the Earth candidate, angular momentum deficit of the system (AMD), mass in embryos stranded in the asteroid belt, and the number of asteroids left. 